\title{
Solar transition region above sunspots ${ }^{\star}$
}

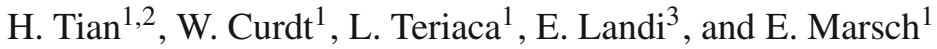 \\ 1 Max-Planck-Institut für Sonnensystemforschung, Max-Planck-Str. 2, 37191 Katlenburg-Lindau, Germany \\ e-mail: tianhui924@gmail.com \\ 2 School of Earth and Space Sciences, Peking University, PR China \\ 3 Naval Research Laboratory, Washington D.C., USA
}

Received 19 March 2009 / Accepted 10 June 2009

\begin{abstract}
Aims. We study the transition region (TR) properties above sunspots and the surrounding plage regions, by analyzing several sunspot reference spectra obtained by the SUMER (Solar Ultraviolet Measurements of Emitted Radiation) instrument in March 1999 and November 2006.

Methods. We compare the SUMER spectra observed in the umbra, penumbra, plage, and sunspot plume regions. The hydrogen Lyman line profiles averaged in each of the four regions are presented. For the sunspot observed in 2006, the electron densities, differential emission measure (DEM), and filling factors of the TR plasma in the four regions are also investigated.

Results. The self-reversals of the hydrogen Lyman line profiles are almost absent in sunspots at different locations (at heliocentric angles of up to $49^{\circ}$ ) on the solar disk. In the sunspot plume, the Lyman lines are also not reversed, whilst the lower Lyman line profiles observed in the plage region are obviously reversed, a phenomenon found also in the normal quiet Sun. The TR densities of the umbra and plume are similar and one order of magnitude lower than those of the plage and penumbra. The DEM curve of the sunspot plume exhibits a peak centered at $\log (T / \mathrm{K}) \sim 5.45$, which exceeds the DEM of other regions by one to two orders of magnitude at these temperatures. We also find that more than 100 lines, which are very weak or not observed anywhere else on the Sun, are well observed by SUMER in the sunspot, especially in the sunspot plume.

Conclusions. We suggest that the TR above sunspots is higher and probably more extended, and that the opacity of the hydrogen lines is much lower above sunspots, compared to the TR above plage regions. Our result indicates that the enhanced TR emission of the sunspot plume is probably caused by a large filling factor. The strongly enhanced emission at TR temperatures and the reduced continuum ensure that many normally weak TR lines are clearly distinctive in the spectra of sunspot plumes.
\end{abstract}

Key words. Sun: UV radiation - Sun: transition region - sunspots - line: profiles

\section{Introduction}

The solar transition region (TR, between $\sim 10^{4} \mathrm{~K}$ and $10^{6} \mathrm{~K}$ ) is the interface between the chromosphere and corona, where the temperature and density change dramatically. Most of the TR emission occurs in the VUV (vacuum ultraviolet) range of the electromagnetic radiation (Wilhelm et al. 2007). Thus, ultraviolet emission lines can provide ample information about the magnetic structures and plasma properties of the TR.

Earlier ultraviolet observations such as those made by the Naval Research Laboratory (NRL) S082-B EUV spectrograph onboard the Skylab space station (Bartoe et al. 1977), and NRL High-Resolution Telescope Spectrograph (HRTS) flown on some rockets and Spacelab2 (Brueckner et al. 1977; Brueckner \& Bartoe 1983; Brueckner et al. 1986), provided much valuable information about the TR. These earlier results were reviewed by Mariska (1992).

Our knowledge of the TR has been enhanced greatly since the SUMER (Solar Ultraviolet Measurements of Emitted Radiation) instrument (Wilhelm et al. 1995; Lemaire et al. 1997) onboard SOHO (Solar and Heliospheric Observatory) began to observe in 1996. Because of its high spectral, spatial, and temporal resolutions, and the wide wavelength coverage, many more TR line profiles than in the past were obtained, identified, and

* Tables 5 and 6 are only available in electronic form at http://www . aanda.org used in intensive studies (Curdt et al. 2001, 2004). In addition, the CDS (Coronal Diagnostic Spectrometer) instrument (Harrison et al. 1995) onboard SOHO has also increased significantly our understanding of the TR structures and dynamics. For a review of these recent progresses, we refer to Wilhelm et al. (2007).

Gabriel (1976) proposed a magnetic network model, in which the TR emission originates in funnels diverging with height from the underlying supergranular boundary. A decade later, Dowdy et al. (1986) proposed a modified model in which only a fraction of the network flux opens, in the shape of funnels, into the corona, while the remainder of the network is occupied by a population of low-lying loops with lengths less than $10 \mathrm{Mm}$. Based on SUMER observations, Peter (2001) suggested a new picture for the structure of the TR, in which the funnels are either connected to the solar wind or just the legs of large loops.

All the models mentioned above refer to the average structures in the TR. However, the solar atmosphere is very inhomogeneous and characterized by different large and small-scale structures. Moreover, studies have shown that the TR is not thermally stratified but strongly nonuniform and magnetically structured (Feldman 1983, 1987; Marsch et al. 2006). The SUMER instrument is well suited to study the difference in TR structures in different regions of the Sun. By combing the technique of magnetic field extrapolation with SUMER observations, it has 
Table 1. Observational parameters of the 5 reference spectra of sunspots.

\begin{tabular}{cccccccccc}
\hline \hline Year & Date & Start time & End time & Detector & Slit & Exposure time $(\mathrm{s})$ & $x\left(^{\prime \prime}\right)$ & $y\left(^{\prime \prime}\right)$ & $\theta^{\circ}$ \\
\hline 1999 & March 16 & $13: 09$ & $16: 14$ & A & 6 & 90 & 44 & -351 & 22 \\
& March 17 & $19: 48$ & $22: 44$ & B & 7 & 90 & 302 & -340 & 28 \\
& March 18 & $17: 35$ & $20: 30$ & B & 7 & 90 & 479 & -360 & 38 \\
& March 19 & $13: 58$ & $16: 54$ & B & 7 & 90 & 627 & -350 & 49 \\
2006 & November 13/14 & $23: 58$ & $02: 52$ & B & 7 & 90 & -78 & -122 & 9 \\
\hline
\end{tabular}

been found that the TR in coronal holes is higher and more extended than in the quiet Sun (Tu et al. 2005a,b; Tian et al. 2008a,b).

An active region is an area with an especially strong magnetic field, where sunspots and plages are frequently formed. Our current empirical knowledge and physical understanding of sunspots were reviewed by Solanki (2003). The sunspot spectra obtained by SUMER reveal some distinct properties (Curdt et al. 2001). For instance, some spectral lines which are not observed in other areas of the Sun stick out in the sunspot spectra. Moreover, in contrast to the quiet Sun, the hydrogen Lyman line profiles in the sunspot are not reversed. Until now, little has been done to understand these phenomena.

Spectral lines with formation temperatures between $\sim 10^{5} \mathrm{~K}$ and $10^{6} \mathrm{~K}$ (upper-TR) often have significantly enhanced intensities at locations overlying sunspot umbrae (Foukal et al. 1974). These features are usually termed sunspot plumes (Foukal 1976). A sunspot plume usually has one end point anchored in the umbra and the other can reach far from the sunspot. It is regarded as nothing more than the common footpoints of several active region loops (Dammasch et al. 2008). Although sunspot plumes have been studied extensively, the reason why the plume emission is so prominent at upper-TR temperatures is still unknown.

Some work has been done to study the plasma properties of sunspot plumes. The electron density, $\log \left(N_{\mathrm{e}} / \mathrm{cm}^{-3}\right)$, of the TR plasma in sunspot plumes is about 10 (Doyle et al. 1985; Doyle \& Madjarska 2003). The sunspot plumes seem to be associated with downflows of TR plasma (Foukal 1976; Brynildsen et al. 2001; Marsch et al. 2004; Brosius \& Landi 2005; Dammasch et al. 2008). The emission measure (EM) curve based on S-055 spectra of sunspot plumes revealed two peaks at $\log (T / \mathrm{K})=5.6$ and 6.1, respectively (Noyes et al. 1985), while a more recent study showed that the plume's differential emission measure (DEM) exhibited only one peak centered at $\log (T / \mathrm{K})=5.6$ or 5.8 (Brosius \& Landi 2005). More work is needed to compare the properties of sunspot plumes and other regions.

In this paper, we present a more complete analysis, by analyzing more sunspot spectra obtained with SUMER at different locations of the Sun, and compare the Lyman line (of main quantum number $n$ higher than 2) profiles, electron densities, DEM curves, and filling factors of the sunspot plume, umbra, penumbra, and the surrounding plage regions. Our results have important physical conclusions for the TR properties above the sunspot and the surrounding plage region.

\section{Observation and data reduction}

We selected five reference spectra of two sunspots observed by SUMER. One sunspot was observed at different locations of the solar disk during March 16-19, 1999. The reference spectrum of the other sunspot was obtained between 23:58 on November 13 and 02:52 of the next day in 2006. The observational details are listed in Table 1 . The pointing in $x$ and $y$ is given in arcseconds and refers to the slit center at the central time of the observation. $\theta$ is the heliocentric angle.

The standard procedures for correcting the SUMER data were applied, including local-gain correction, dead-time correction, flat-field correction, and image destretching.

In the earlier work of Curdt et al. (2000), the 1999 sunspot was analyzed. However, only the reference spectrum obtained on March 18 was used. This spectrum was also used to produce the sunspot atlas, and both the context image and slit position can be found in Curdt et al. (2001). Here we extend the earlier work by analyzing four reference spectra of this sunspot obtained at different solar locations. Because of the solar rotation, this sunspot rotated from the central meridian towards the limb, and its heliocentric angle increases from $22^{\circ}$ to $49^{\circ}$. Thus, we can study the center to limb variation in the Lyman line profiles of the sunspot, as shown in Fig. 1.

The sunspot observed in 2006 was large in size, and several reference spectra for it were obtained, all near the disk center. For this reason, we analyze only one reference spectrum of this sunspot. During this observation, TRACE (Transition Region and Corona Explorer) obtained an image of the $1600 \AA$ passband at 00:32 on November 14, 2006. The sunspot image observed by TRACE is shown in panel (A) of Fig. 2. Also shown there is the approximate location of the SUMER slit. The SUMER instrument also scanned this sunspot region by using several typical TR lines (N IV, O IV, Si IV, O III), and the relevant results were published in Teriaca et al. (2008). Although the scans were completed at different times, and a temporal variation may have occurred in the sunspot region, their Fig. 1 still provides an impression of the TR emission from the sunspot and the surrounding plage regions.

\section{Hydrogen Lyman line profiles}

Hydrogen is the most abundant element in the solar atmosphere and its resonance lines play an important role in the overall radiative energy transport of the Sun (Fontenla et al. 1988). Profiles of hydrogen Lyman series can also be used to diagnose nonthermal effects in solar flares (Fang et al. 1995; Xu et al. 2005).

Line profiles of the full hydrogen Lyman series can be acquired by SUMER at high spectral resolution. It has been found that the average profiles for Ly- $\beta(n=2)$ through $\mathrm{Ly}-\epsilon(n=5)$ are self-reversed and stronger in the red horns, while the higher $\mathrm{H}$ Lyman series lines (from $\mathrm{Ly}-\zeta$ to $\mathrm{Ly}-\lambda,(n=6, \cdots, 11)$ ) are flat-topped (Warren et al. 1998; Xia 2003; Xia et al. 2004). Higher Lyman lines obtained near the limb were analysed by Marsch et al. (1999) and Marsch et al. (2000), in which the authors found an increase in the line width with decreasing main quantum number and an unexpectedly flat hydrogen-temperature gradient. The Ly $-\alpha$ profiles in the quiet Sun were obtained by SUMER through several non-routine observations, by closing the aperture door of SUMER to reduce the incoming photon flux to a 20\%-level (Curdt et al. 2008; Tian et al. 2009). The average $\mathrm{Ly}-\alpha$ profile was found to be strongly reversed and have 

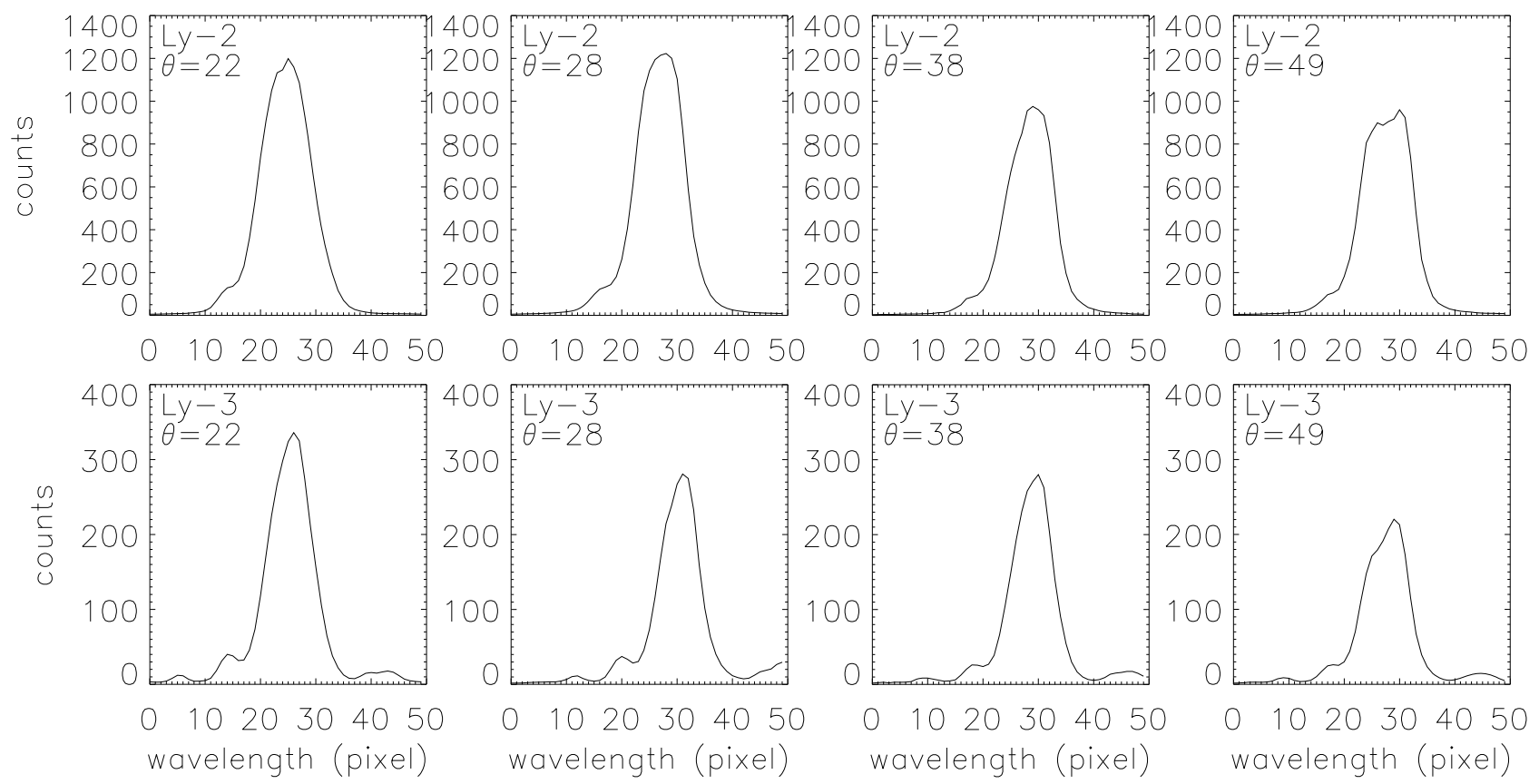

Fig. 1. Averaged profiles of Ly-2 (upper panels) and Ly-3 (lower panels) in the sunspot observed on 16th $\left(\theta=22^{\circ}\right), 17$ th $\left(\theta=28^{\circ}\right), 18$ th $\left(\theta=38^{\circ}\right)$, and 19th $\left(\theta=49^{\circ}\right)$ of March in 1999. $\theta$ is the heliocentric angle.

(A)

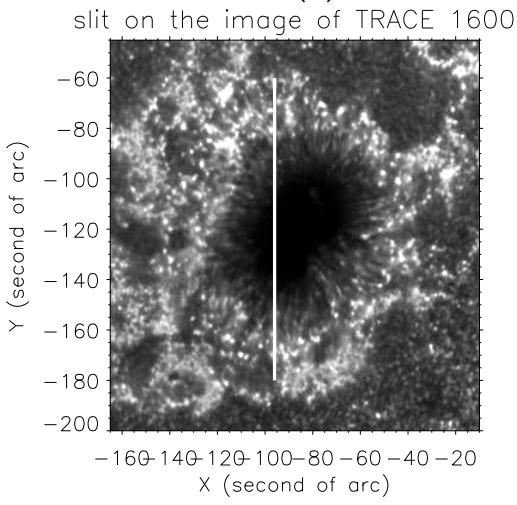

(B)

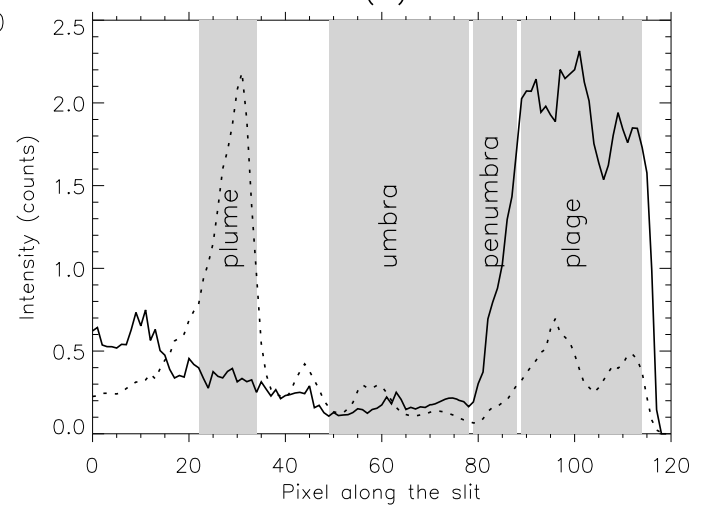

Fig. 2. The sunspot observed on 14th of November, 2006. (A) The SUMER slit is located on the image of the $1600 \AA$ passband of TRACE. The TRACE image was obtained at 00:32 on November 14, 2006. (B) The intensities of continuum around $1045 \AA$ (solid line) and O VI (1031.9 , dotted line) along the slit. Four segments corresponding to the plage, penumbra, umbra, and sunspot plume are marked in grey. The O VI intensity has been divided by 600 . a stronger blue horn. It is believed that the opposite asymmetries in the average profiles of Ly- $\alpha$ and higher Lyman lines are probably caused by the combined effect of flows in the different layers of the solar atmosphere and opacity differences of the lines (Gunár et al. 2008; Tian et al. 2009).

The Lyman series are important for diagnosing the variation in the thermodynamic conditions in prominences (Vial 2007). Prominence thread models including multi-level non-LTE transfer calculations have shown that the profiles of Lyman lines are more reversed when seen across than along the magnetic field lines (Heinzel et al. 2005). This behaviour was confirmed in a prominence observation by Schmieder et al. (2007).

In sunspot regions, the Lyman line profiles exhibit properties that are different from the average profiles. The sunspot atlas of SUMER reveals that the Lyman line profiles observed in the sunspot are almost not reversed (Curdt et al. 2001). However, the authors did not mention this phenomenon in that paper. Here we extend this earlier work by analyzing four reference spectra of this sunspot obtained at different solar locations. Since the magnetic field lines in sunspots are almost vertical, we can use the 4 spectra to study the properties of the Lyman line profiles in sunspots and the dependence of their self-reversals on the orientation of field lines.

The Lyman line profiles in the four spectra are all not or only slightly reversed, similar to those in off-limb coronal hole observations (Marsch et al. 2000). This result suggests that the TR plasma of the sunspot is almost optically thin, regardless of the location where the sunspot is observed. Since it is known that the higher order Lyman lines are optically thinner than lower order Lyman lines, radiative transfer effects, if present, should be more pronounced in lower order Lyman lines than in higher order Lyman lines. Thus, we present only the Ly-2 and Ly-3 profiles averaged over the sunspot (umbra) portion of the slit in Fig. 1. Note that there are some blends with the Lyman lines (Curdt et al. 2001). From an inspection of Fig. 1, it seems that profiles begin to reverse when the vertical field lines of the sunspot make an angle of about $49^{\circ}$ with respect to the line of sight. This phenomenon seems to be similar to the observational result for prominences, namely that the Lyman profiles are more reversed when seen across the field lines than along the field lines (Schmieder et al. 2007). However, from $\theta=22^{\circ}$ to $\theta=38^{\circ}$, it is difficult to say whether or not there is a trend in the shapes of the top parts of the profiles. 

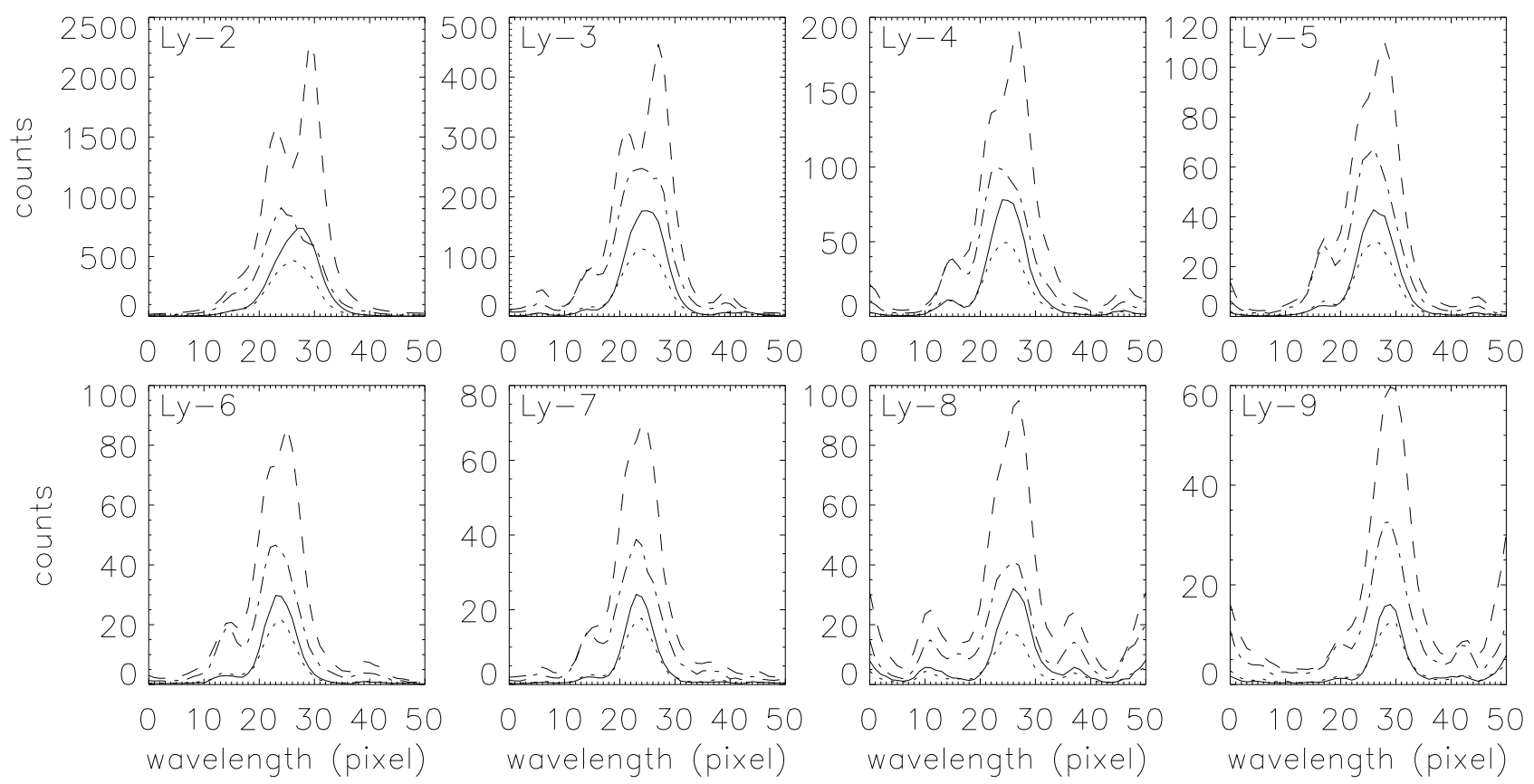

Fig. 3. The dashed, dot-dashed, dotted, and solid lines represent the average profiles of the Lyman lines (from Lyman-2 to Lyman-9) observed in the plage, penumbra, umbra, and plume for the 2006 data set. Note that the profiles of the umbra were multiplied by a factor of two.

The sunspot observed in 2006 is a large one, and the SUMER slit also caught part of a sunspot plume and the surrounding plage region. Figure 2 shows the intensities of the continuum around $1045 \AA$ and of the O VI (1031.9 $\AA$ ) line along the slit, and the resulting curves clearly show the umbra, penumbra, and plage regions as well as part of the sunspot plume. In this paper, the term intensity is regarded as being equivalent to radiance. The sunspot plume shows enhanced radiance in typical TR lines, which corresponds to the slit segment with the highest intensities of $\mathrm{O}$ VI in our reference spectra. We selected four portions of the slit, which are marked in grey in Fig. 2 and correspond to the umbra, penumbra, plage, and plume locations. The average Lyman line profiles (from Ly-2 to Ly-9) of the four parts are presented in Fig. 3. For the blends with the Lyman lines, we refer to Curdt et al. (2001).

Our results show that the self-reversals of all the hydrogen Lyman line profiles are absent in the umbra and plume regions. The lower Lyman line profiles observed in the plage region are obviously reversed, a phenomenon similar to the normal quiet Sun (one can refer to the atlas presented in Curdt et al. 2001). The profiles in the penumbra are not so peaked as those in the umbra and plume, and not so reversed as those in the plage. We also note that the Lyman line profiles observed in the plage have a very strong red-horn asymmetry (the red horn is much stronger than the blue horn), which might be at least partly caused by the strong red shift observed in the TR. The asymmetries of the lower Lyman line profiles in the penumbra seem to be opposite to those of the plage, which might indicate a different pattern of flows in the upper atmosphere of the two regions.

Unfortunately, so far SUMER has not been used to complete Ly $-\alpha$ observations of the sunspot. We note that Fontenla et al. (1988) presented a Ly- $\alpha$ profile (see Fig. 12 in the paper) of a sunspot observed by the Ultraviolet Spectrometer and Polarimeter on the SMM (Solar Maximum Mission) spacecraft. As mentioned in that paper, the emission suffered from geocoronal absorption. After correcting for this effect, their Ly $-\alpha$ profile seems to be flat-topped, which is very different from the strongly reversed Ly $-\alpha$ profiles in the quiet Sun (Curdt et al. 2008; Tian et al. 2009).

The above results indicate that the opacity is strongly reduced above the sunspot, with respect to the surrounding plage region. By analysing the emission lines of $\mathrm{H}_{2}$ in the sunspot as well as the quiet Sun, Jordan et al. (1978) and Bartoe et al. (1979) concluded that the opacity over the sunspot is about an order of magnitude lower than in the quiet Sun. The different opacities above sunspots and plages are confirmed by the intensity ratio between $\mathrm{Ly}-\alpha$ and $\mathrm{Ly}-\beta$, which is about 200 in the sunspot umbra and 130 in the plage. In the sunspot, the profiles are only weakly absorbed. In the plage, the opacity is higher and thus the absorption is enhanced. Since Ly $-\alpha$ has a larger opacity, its absorption will be stronger than Ly- $\beta$, which will lead to a lower observed value of the intensity ratio. However, we note that the Ly $-\alpha$ line was recorded on the attenuator, so the measurement of its intensity is highly uncertain and we do not consider it in detail here.

Since the Lyman line profiles in the plage are quite similar to those observed in the normal quiet Sun, we may expect a similar opacity in both regions. This similarity might be the result of the similar magnetic structures. In both regions, magnetic loops reaching different heights are the dominant structures. The emission sources of Lyman lines are located mainly in loops that reach the layers of chromosphere and TR. When the chromospheric Lyman line photons travel through the upper atmosphere, they will be initially absorbed by the hydrogen atoms in upper-chromospheric and TR loops, and further absorbed in coronal loops. Since the density decreases and the temperature increases with height above the temperature minimum, there are more hydrogen atoms, and the absorption is much stronger in the upper chromosphere and lower-TR layers than at higher layers. In the plage region and quiet Sun, the Lyman line emission originates by a large fraction in the chromosphere and are strongly absorbed in the upper chromosphere and lower TR, leading to a strong absorption at the center of the Lyman line profiles.

The scenario seems to differ in sunspot regions. The almost Gaussian-shaped profiles suggest a weak absorption of 
Table 2. Electron density $\left(\log \left(N_{\mathrm{e}} / \mathrm{cm}^{-3}\right)\right)$ measurements, for the sunspot observed in 2006.

\begin{tabular}{lrlllll}
\hline \hline Ion & Wavelength pair $(\AA)$ & $T_{\mathrm{f}}(\log \mathrm{K})$ & Umbra & Penumbra & Plage & Plume \\
\hline Si III & $1301.16 / 1298.96$ & 4.68 & $11.1_{-0.4}^{+0.5}$ & $>11.0$ & & $11.1_{-0.4}^{+0.5}$ \\
C III & $1175.98 / 1175.24$ & 4.84 & $>9.3$ & $>9.3$ & $>9.2$ & $>9.4$ \\
O IV & $1399.77 / 1401.16$ & 5.18 & $10.2_{-1.2}^{+0.4}$ & $11.0_{-0.5}^{+0.7}$ & $10.8_{-0.5}^{+0.5}$ & $10.3_{-0.3}^{+0.4}$ \\
O IV & $1407.39 / 1401.16$ & 5.18 & $10.3_{-0.8}^{+0.4}$ & $10.9_{-0.4}^{+0.5}$ & $11.0_{-0.4}^{+0.6}$ & $10.4_{-0.7}^{+0.4}$ \\
O v & $758.68 / 761.13$ & 5.37 & $10.1_{-0.2}^{+0.1}$ & $10.9_{-0.2}^{+0.3}$ & $11.0_{-0.3}^{+0.3}$ & $10.1_{-0.1}^{+0.2}$ \\
O v & $759.43 / 761.13$ & 5.37 & $10.0_{-0.1}^{+0.2}$ & $11.0_{-0.2}^{+0.5}$ & $11.0_{-0.2}^{+0.5}$ & $10.1_{-0.2}^{+0.1}$ \\
O v & $760.43 / 761.13$ & 5.37 & $10.0_{-0.2}^{+0.2}$ & $11.2_{-0.3}^{+0.6}$ & $11.0_{-0.3}^{+0.3}$ & $10.1_{-0.2}^{+0.2}$ \\
O V & $761.99 / 761.13$ & 5.37 & $10.0_{-0.1}^{+0.2}$ & $10.9_{-0.2}^{+0.4}$ & $11.0_{-0.2}^{+0.4}$ & $10.0_{-0.1}^{+0.2}$ \\
Mg VIII & $769.38 / 782.34$ & 5.90 & $<12.0$ & & & $<11.9$ \\
Fe XII & $1349.43 / 1241.95$ & 6.13 & $9.3_{-1.3}^{+1.3}$ & $<10.1$ & $<10.2$ & $<7.6$ \\
\hline
\end{tabular}

the Lyman line emission. This observational result seems to favor a scenario where there is less chromospheric plasma above sunspots, which might be the case because the sunspot is much cooler than the surrounding regions. In this case, the ratio of the chromospheric to TR contributions of the Lyman line radiation is lower in sunspots than in plage regions. As a result, the chromospheric emission will only be weakly absorbed because there is little absorbing material in the upper chromosphere. Moreover, the absorption of the TR emission is weak because of the lower density above the emission sources.

Sunspot plumes often show greatly enhanced emission at upper-TR temperatures $\left(\sim 10^{5}\right.$ and $\left.10^{6} \mathrm{~K}\right)$, while at lower or higher temperatures the emission is rather weak (e.g., Foukal et al. 1974; Brosius \& Landi 2005). Sunspot plumes are frequently reported to be associated with TR downflows (e.g., Marsch et al. 2004; Dammasch et al. 2008). Observational results seem to reveal that plasmas at TR temperatures dominate in plumes. Thus, the ratio of TR to chromospheric contributions to the Lyman line emissions may be significantly higher in plumes. These plume loops are large in size (and far reaching) and the overlying corona is insufficiently dense to cause an obvious dip at the center of the Lyman line profiles.

The above explanations are based mainly on a static stratified atmosphere. However, it is well known that the real solar atmosphere, especially the TR over sunspots, is rather inhomogeneous and dynamic (Brynildsen et al. 1999a,b). It has been found that the Lyman line profiles can be modified by TR flows in the quiet Sun (Curdt et al. 2008; Tian et al. 2009). In sunspot regions, both significant upflows and downflows have been frequently reported (e.g., Kjeldseth-Moe et al. 1988; Teriaca et al. 2008). Thus, we should not exclude the possibility that additional effects, such as the presence of velocities and radiation penetrating from the sides in the inhomogeneous plasma, may alter the source functions of the Lyman lines and also affect the Lyman line profiles.

\section{Electron densities}

Based on the assumption that the plasma is in ionization equilibrium, one can calculate the electron density from line-ratio observations (for a review, see Xia 2003; Wilhelm et al. 2004). Density-sensitive line pairs usually include two emission lines within the same ion. In the de-excitation process, the relative importance of radiative decay to collisional de-excitation is different for the two lines.

There are several density-sensitive line pairs in the SUMER spectral range (Wilhelm et al. 1995). However, some of these lines are either severely blended with other lines and difficult to decompose, or too weak for a reliable calculation. We selected as many lines as possible to calculate the densities for the sunspot observed in 2006. All of these line pairs have a weak dependence on the electron temperature. Fortunately, the few densitysensitive lines are close in wavelength, so that the effects of temporal variations on the measured densities are minimized. By applying the method of single- or multi-Gaussian fitting to the profiles of these lines averaged in the plage, umbra, penumbra, and plume, we were able to obtain the intensities. The theoretical relations between intensity ratios of line pairs and electron densities were taken from the CHIANTI data base (Dere et al. 1997; Landi et al. 2006). The details of the line pairs and density calculations are listed in Table 2 . Here $T_{\mathrm{f}}$ represents the formation temperature of the ion. We attempted to measure the electron density in all temperature regimes, but accurate measurements could only be carried out for the lower-temperature region; the available coronal lines allow us to derive only broad estimates. We assume a $15 \%$ uncertainty in the determination of the line intensities. This error finally propagates into the uncertainties for the densities listed in Table 2.

The most accurate measurements are obtained in the transition region from O IV and OV. The O IV $1401.16 \AA$ line is blended by the chromospheric line S I $1401.51 \AA$, and the O IV $1407.39 \AA$ line is blended with two second order O III lines (703.845 $\AA, 703.85 \AA$ ). However, both of these blends can be resolved by a two-component Gaussian fitting, and thus we are able to obtain reliable intensities of the O IV $1407.39 \AA$ and O IV $1401.16 \AA$ lines.

The electron densities derived by using $\mathrm{O} \mathrm{V}$ line pairs show a general consistency. In the plage and penumbra regions, the value of $\log \left(N_{\mathrm{e}} / \mathrm{cm}^{-3}\right)$ is around 11 , which is one order of magnitude larger than in the umbra and plume regions. The derived density in the sunspot plume here is consistent with those derived by Doyle et al. (1985) and Doyle \& Madjarska (2003). In Doyle et al. (1985), the authors used the ratio of O v $760 \AA / 630 \AA$ obtained by the S-055 EUV spectrometer onboard Skylab and derived a density of $\log \left(N_{\mathrm{e}} / \mathrm{cm}^{-3}\right)=10$. Doyle \& Madjarska (2003) measured the density of the sunspot plumes observed by SUMER on March 18, 1999. By using the same $\mathrm{O} \mathrm{V}$ line pairs as we did, they obtained a density of $\log \left(N_{\mathrm{e}} / \mathrm{cm}^{-3}\right)=9.9$. The $\mathrm{O}$ V 759.43 line is suggested to be blended by S IV 759.34 (Curdt et al. 2001). However, the measurement of Doyle \& Madjarska (2003) indicates that this blend should not be significant. Our result confirms this finding.

The average umbra and plume densities derived by using the $\mathrm{O}$ IV line pairs seem to be higher than in the $\mathrm{O} V$ results. 
However, the differences are within the uncertainties. We found that the intensity of the plume part seen in O IV is not as strong as that seen in $\mathrm{O} v$, which indicates that the strongest part of the plume emission might not have been caught by the slit, or that the plume was less prominent at the observation time of the $\mathrm{O}$ IV line pairs. By using the ratio of O IV $625 \AA / 790 \AA$ from the S055 sunspot plume spectrum, Doyle et al. (1985) derived a density of $\log \left(N_{\mathrm{e}} / \mathrm{cm}^{-3}\right)=10.3$, which is very close to our plume density at the same temperature. However, by using the line pair of O IV $625.8 \AA / 554.5 \AA$ measured by CDS, Brosius \& Landi (2005) derived relatively low values $\left(\log \left(N_{\mathrm{e}} / \mathrm{cm}^{-3}\right)=9.6 \sim 9.7\right)$ of plume density. However, the line of O IV $625.8 \AA$ was rather weak and blended with $\mathrm{Mg}$ X $624.9 \AA$ in the red wing, so the derived densities were highly uncertain.

The C III and Si III measurements only provide lower limits to the electron density. The C III values agree with the TR values from the oxygen ions, while Si III infers a higher density than the oxygen ions in the umbra and plume. This might suggest the presence of a significant density gradient in the umbra and in the plume from the chromosphere to the lower TR, which would indicate a decrease in the plasma pressure, if we calculate the pressure to be $p=N_{\mathrm{e}} T_{\mathrm{f}}$. However, possible strong temperature gradients on one side, and opacity effects in the C III and Si III on the other, cast huge uncertainties on the presence of a density and pressure gradient in the umbra and the plume.

In the corona, $\mathrm{Mg}$ VIII and Fe XII provide only very rough estimates of or upper limits to the electron densities, which are consistent with almost any value measured in the corona in quiescent conditions. In this case, our SUMER dataset is unable to provide significant constraints.

The umbra and plume densities derived here are similar to, or slightly larger than, the density of the normal quiet Sun, which has an upper $\operatorname{limit}$ of $\log \left(N_{\mathrm{e}} / \mathrm{cm}^{-3}\right)=9.87$ at $\log (T / \mathrm{K})=5.25 \mathrm{in}$ Griffiths et al. (1999). A similar result is also obtained by Brosius \& Landi (2005), in which the densities of plumes and quiet Sun areas are estimated to be $\operatorname{around} \log \left(N_{\mathrm{e}} / \mathrm{cm}^{-3}\right)=9.6 \sim 9.7$ and $\log \left(N_{\mathrm{e}} / \mathrm{cm}^{-3}\right)=9.4$, respectively, by using the same line pair of O IV $625.8 \AA / 554.5 \AA$ as observed by the CDS instrument.

By analyzing the EUV data obtained by the Harvard spectrometer on the Apollo telescope mount, Foukal et al. (1974) measured a significant decrease in the gas density of the umbra relative to the surrounding plage. Here we confirm this result by finding that the densities of the umbra and plume at TR temperatures are about a factor of 10 lower than of the plage. Our measurements seem to indicate that the sunspot plasma emitting at TR temperatures is higher and probably more extended than in the surrounding plage region. Since the density of the solar atmosphere decreases almost exponentially with height above the photosphere, this scenario naturally leads to a much lower density in the TR above sunspots, as compared to the TR above the surrounding plage. This scenario also implies that the sunspot TR temperature is much lower than the surrounding temperature at the same heights. Our scenario predicts a temperature structure of sunspots as that proposed by Nicolas et al. (1982). Our conclusion is also consistent with that of Guo et al. (2009), in which the authors suggested that stronger magnetic fields correspond to higher formation heights of VUV lines.

We note that this TR scenario is similar to that for a coronal hole, since the TR in the coronal hole is also found to be higher and more extended than the TR in the quiet Sun (Tu et al. $2005 \mathrm{a}, \mathrm{b}$; Tian et al. 2008a,b). The average Ly- $\beta$ and Ly-3 profiles in the coronal hole atlas are not reversed, a phenomenon similar to the sunspot, whilst they are obviously reversed in the quiet Sun (Curdt et al. 2001). Moreover, solar wind flows out along magnetic funnels in coronal holes (Tu et al. 2005a; Esser et al. 2005), and the signature of upflows associated with open field lines was also found in sunspots (Marsch et al. 2004). These results infer a similarity between the properties of the TR above sunspots/plages and the TR in $\mathrm{CH} / \mathrm{QS}$ (coronal hole and quiet Sun).

It was suggested that the downflow of TR plasma is essential to the existence of plumes (Brynildsen et al. 2001; Foukal 1976). However, it remains debated whether this is achieved by siphon flows along far reaching loops (Brynildsen et al. 2001; Doyle \& Madjarska 2003; Brosius 2005) or by cooling and condensing coronal plasma falling downward along the "cold surface" of the plume (Noyes et al. 1985; Brosius \& Landi 2005; Dammasch et al. 2008). A siphon flow is possibly driven by a strong asymmetric heating and the resulting pressure difference in two loop legs (McClymont \& Craig 1987; Mariska 1988; Spadaro et al. 1991). This type of flow may cause a blue shift in one leg and red shift in the other. Brynildsen et al. (2001) concluded that the inflow of plasma at TR temperatures from locations well outside the sunspots is a necessary requirement for the sunspot plume to occur. This hypothesis seems to be supported by the observational result of Brosius (2005), in which a significantly higher density $\left(\log \left(N_{\mathrm{e}} / \mathrm{cm}^{-3}\right)=9.8\right.$, compared to $\log \left(N_{\mathrm{e}} / \mathrm{cm}^{-3}\right)=8.9$ in the plume, in an upflow region outside the sunspot was observed by CDS. Doyle \& Madjarska (2003) also measured higher densities (with $\log \left(N_{\mathrm{e}} / \mathrm{cm}^{-3}\right)$ ranging from 10.20 to 10.45 ) in nearby quiet regions (their $Q S_{1}$ might be a plage region) than in the plume $\left(\log \left(N_{\mathrm{e}} / \mathrm{cm}^{-3}\right) \sim 9.9\right)$, and suggested that the gas pressure difference might be sufficient to drive siphon flows from outside the spot into the umbra. Our measured densities in different regions also seem to support this idea. It is generally believed that sunspot plumes are associated with loops in which one leg is anchored in the umbra and the other anchored outside the sunspot, probably in the plage region. Our measurement shows that the densities at TR temperatures are one order of magnitude lower in the umbra and plume than in the plage. This pressure difference is certainly sufficient to initiate a siphon flow in the loop.

\section{Differential emission measure}

\subsection{Method}

The plasma in the upper solar atmosphere can usually be assumed to be optically thin, although this assumption does not hold for the coldest ions observed in the SUMER spectra, such as neutrals and singly ionized species. In the optically thin case, the radiance of a line can be written as

$I_{j i}=\frac{1}{4 \pi} \int G_{j i}\left(T, N_{\mathrm{e}}\right) N_{\mathrm{e}}^{2} \mathrm{~d} h$

where $N_{\mathrm{e}}$ is the electron density, $h$ is the height of the emitting volume along the line of sight, and $G_{j i}\left(T, N_{\mathrm{e}}\right)$ is the line contribution function.

The contribution function includes all the atomic physics involved in the process of line formation, and can be computed as a function of temperature and density using spectral codes that include all the atomic parameters necessary to calculate the contribution function. In the present work we will use version 5.2.1 of the CHIANTI database (Dere et al. 1997; Landi et al. 2006), the ion fraction dataset of Mazzotta et al. (1998), and the photospheric abundances of Grevesse \& Sauval (1998). 

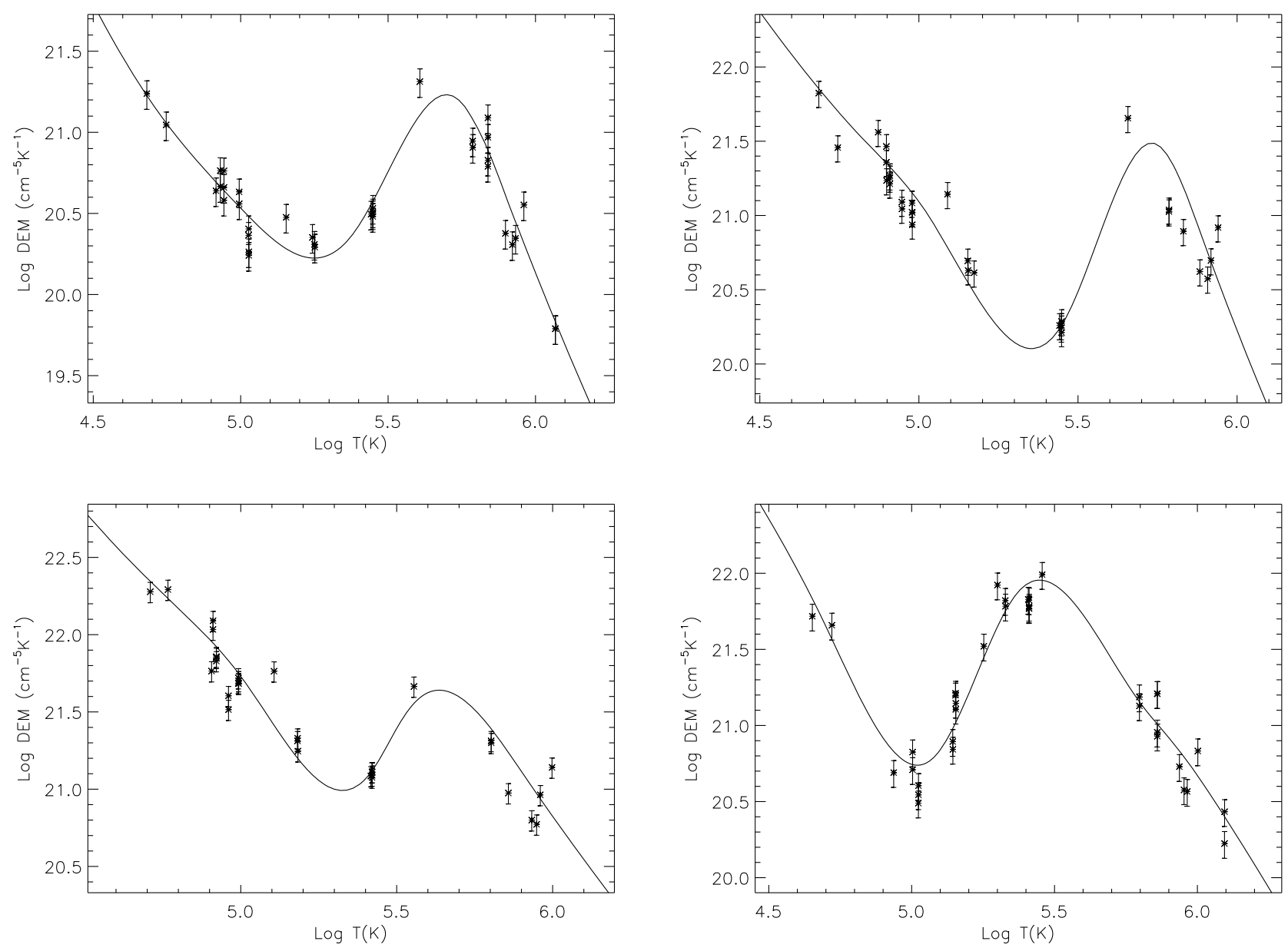

Fig. 4. DEM curves for the four regions. Top left: umbra; top right: penumbra; bottom left: plage; Bottom right: plume.

The $N_{\mathrm{e}}^{2} \mathrm{~d} h$ term beneath the sign of the integral on the righthand side of Eq. (1) includes plasma conditions involved in the process of line formation. It is an important quantity when studying the thermal structure of the solar corona and comparing predictions from theoretical models with observations. When the plasma is multi-thermal and there is a continuous relationship between the amount of plasma and temperature, then Eq. (1) can be rewritten by defining the Differential Emission Measure (DEM) function $\varphi(T)$ as

$$
\begin{aligned}
\varphi(T) & =N_{\mathrm{e}}^{2} \frac{\mathrm{d} h}{\mathrm{~d} T}, \\
I_{j i} & =\frac{1}{4 \pi} \int G_{j i}\left(T, N_{\mathrm{e}}\right) \varphi(T) \mathrm{d} T,
\end{aligned}
$$

where the DEM indicates the amount of material in the plasma as a function of temperature. Several methods have been developed to determine $\varphi(T)$ from a set of observed lines. Reviews of the main examples of these methods can be found in Harrison \& Thompson (1992) and Phillips et al. (2008). In the present work, we use the iterative technique developed by Landi \& Landini (1997). In this technique, an initial, arbitrary $\varphi_{0}(T)$ curve is first assumed. Corrections to this curve are calculated by evaluating the ratio of observed line fluxes to theoretical values predicted using the $\varphi_{0}(T)$ curve. Each correction $\omega_{0}\left(T_{\text {eff }}\right)$ is associated with an effective temperature $T_{\text {eff }}$ defined as

$$
\log T_{\text {eff }}=\frac{\int G\left(T, N_{\mathrm{e}}\right) \varphi_{0}(T) \log T \mathrm{~d} T}{\int G\left(T, N_{\mathrm{e}}\right) \varphi_{0}(T) \mathrm{d} T} .
$$

A new $\varphi_{1}(T)$ curve is determined by first calculating the values of the new $\varphi_{1}(T)$ curve at the temperatures $T_{\text {eff }}$ to be

$\varphi_{1}\left(T_{\text {eff }}\right)=\varphi_{0}\left(T_{\text {eff }}\right) \times \omega_{0}\left(T_{\text {eff }}\right)$,

and then interpolating the results over temperatures to provide a continuous function. The resulting $\varphi_{1}(T)$ curve is then used as an initial DEM curve in the next iteration to calculate new corrections. The procedure converges when the corrections to the $i-t h$ DEM curve are all unity within the uncertainties. This technique reaches the final solution in just a few iterations, and the final result is independent of the initial arbitrary $\varphi_{0}(T)$ curve.

\subsection{DEM curves}

We adopted an electron density of $\log \left(N_{\mathrm{e}} / \mathrm{cm}^{-3}\right)=10.0$ for the umbra and the plume, and $\log \left(N_{\mathrm{e}} / \mathrm{cm}^{-3}\right)=11.0$ for the penumbra and plage, to calculate the contribution functions to be used for DEM diagnostics. The DEM curves determined using the spectral lines listed in Table 5 are shown in Fig. 4. To obtain 
those curves, it was necessary to complete an additional selection of the spectral lines to be used, as well as to modify the adopted element abundances.

When we applied the DEM diagnostic technique to all the listed lines at once, very large disagreements were found between lines of the same ion, and between lines of different ions with similar $\log T_{\text {eff }}$. These disagreements occurred in all four regions, and made it impossible to calculate a DEM curve for each region because: 1$)$ the correction curves $\omega(T)$ never converged to unity and showed an oscillatory behavior; and 2) even when they were close to unity, the disagreements between different ions and between lines of the same ion were so large that the resulting DEM curve was meaningless.

The causes of these problems are probably numerous: atomic physics problems in the level population calculation within each ion; inaccurate ion abundances; opacity effects (for the coldest lines in the dataset); non-photospheric element abundances; or temporal variability. Opacity effects can be ruled out for all the listed lines, except for the strongest lines belonging to the ions formed at lowest temperatures. The intensities of all other cold lines are too weak to provide significant self-absorption. Moreover, Brooks et al. (2000) ruled out opacity effects for many of the coldest ions, although they showed that there may be significant opacity effects for C II and C III. Atomic physics problems can be ruled out as the discrepancies between lines of the same ions are much larger than any effect of inaccurate collisional or radiative excitation rates. The same can be said for ion abundances, unless non-equilibrium conditions are present in the emitting plasma. The most likely cause of the problems that we encountered is the temporal variability of the plasma. Any temporal changes in the physical properties of the plasma directly affect our DEM results because the SUMER data were taken over several hours, and the transition region plasma is known to be changing even within a few minutes.

To minimize the effects of temporal variability, we restricted our analysis to lines that were emitted within about $20 \mathrm{~min}$. Since SUMER scans its wavelength range by shifting the reference wavelength by fixed amounts from one exposure to the next, the selected time frame in our observations corresponds to $120 \AA$. The optimal spectral range including the highest number of lines and ions in the widest temperature range within $120 \AA$ for detector B is the shortest wavelength range of $670-790 \AA$. To further sample the corona, we also included the two Fe XII lines $1241.95 \AA$ and $1349.43 \AA$, even though they are far apart from the selected interval. This choice makes some sense since coronal lines might experience less temporal variation than the TR ones, so they might still be representative of the corona.

We have applied the DEM diagnostic technique to the lines observed between $670 \AA$ and $790 \AA$ in first order, and this time the solutions converged to the curves displayed in Fig. 4. However, there was one last correction necessary to determine the final DEM curves: the abundances of the low-FIP elements (those with a First Ionization Potential lower than $10 \mathrm{eV}$ ) needed to be increased by a factor of 10 . This huge factor is 2.5 times higher than the the factor required by the normal FIP effect. This correction only affects the corona, since below $\log (T / \mathrm{K})=5.7$ all the ions belong to high-FIP elements; in contrast, all coronal ions except Ne VIII belong to low-FIP elements. This correction was required to ensure agreement between results derived for the $\mathrm{Mg}$ VIII and Ne VIII lines, whose temperatures of formation are very similar. It is important to note that Ne VIII belongs to the lithium isoelectronic sequence. This sequence has been found to overestimate theoretical emissivities relative to those of the
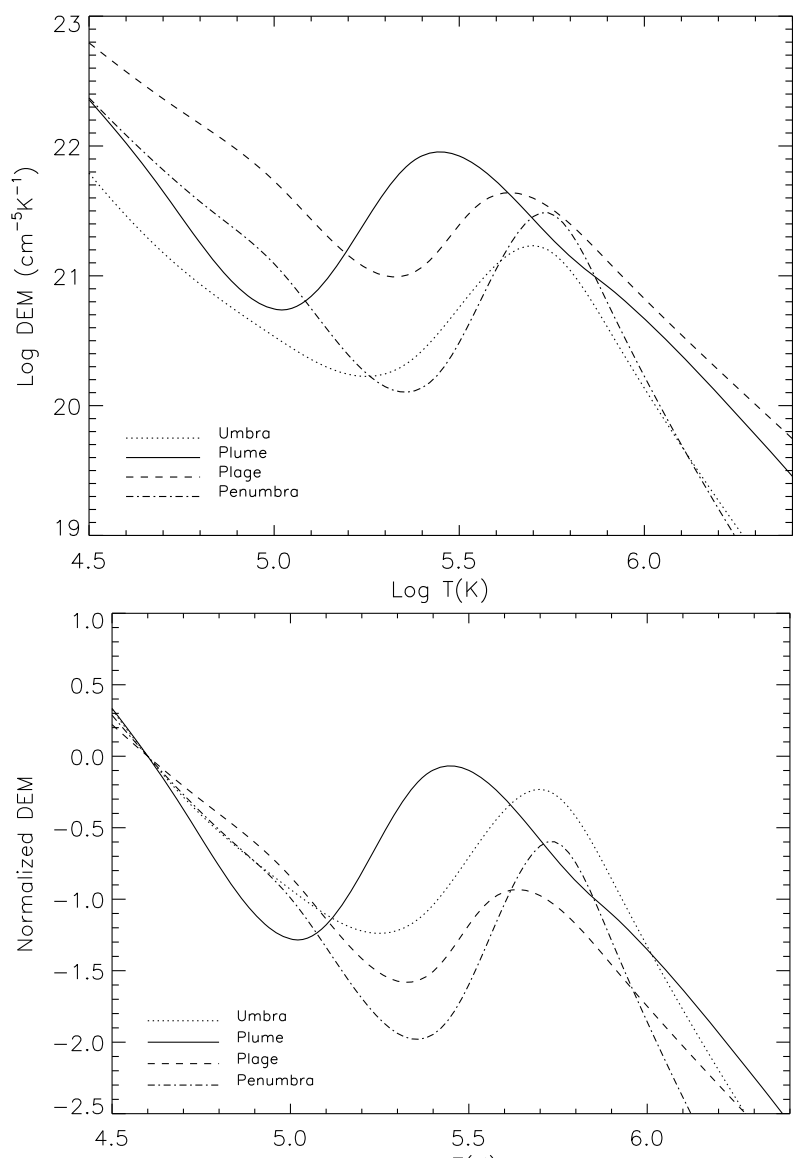

Fig. 5. Comparison of the DEM curves from the four regions. Upper: absolute values; lower: normalized values.

other elements, leading to EM and DEM measurements lower by a factor $\simeq 2$ than those of elements formed at similar temperatures (Dupree 1972; Landi et al. 2002). If we take this systematic effect into account for Ne VIII, the effective increase in the abundance of low-FIP elements is a factor of 5, much closer to the standard FIP effect commonly measured in the corona (i.e., Feldman \& Laming 2000).

All the DEM curves that we measured exhibited an enhanced peak at $\log (T / \mathrm{K}) \simeq 5.6-5.8$, in the upper transition region. The only exception is the plume DEM, which peaks at lower temperatures, $\log (T / \mathrm{K})=5.45$. The peak of the plume DEM curve exceeds the DEM of other regions by one to two orders of magnitude at these temperatures. All the curves are able to reproduce the observed lines, but some scatter is still present among the measurements provided by each line. It is difficult to say whether the DEM curves have a coronal component beyond $\log (T / \mathrm{K})=6.0$, because the restricted dataset of lines we used does not include real coronal lines formed at temperatures higher than one million degrees. The only exceptions are the two Fe XII lines, but their contribution function has a low temperature tail that is strongly influenced by the colder plasma. Its emission measure is so large, that the $\log T_{\text {eff }}$ of the Fe XII lines is lower than the temperature of maximum abundance for Fe XII. The curves that we determined are compared with each other in Fig. 5. In absolute units (upper panel of Fig. 5), the curves are rather similar, the only exception being the plume curve, whose peak is at lower temperatures. When normalized to their value at $\log (T / \mathrm{K})=4.7$, the slope of all four curves is approximately the same up to $\log (T / \mathrm{K})=5.0$. This behavior was noted by 


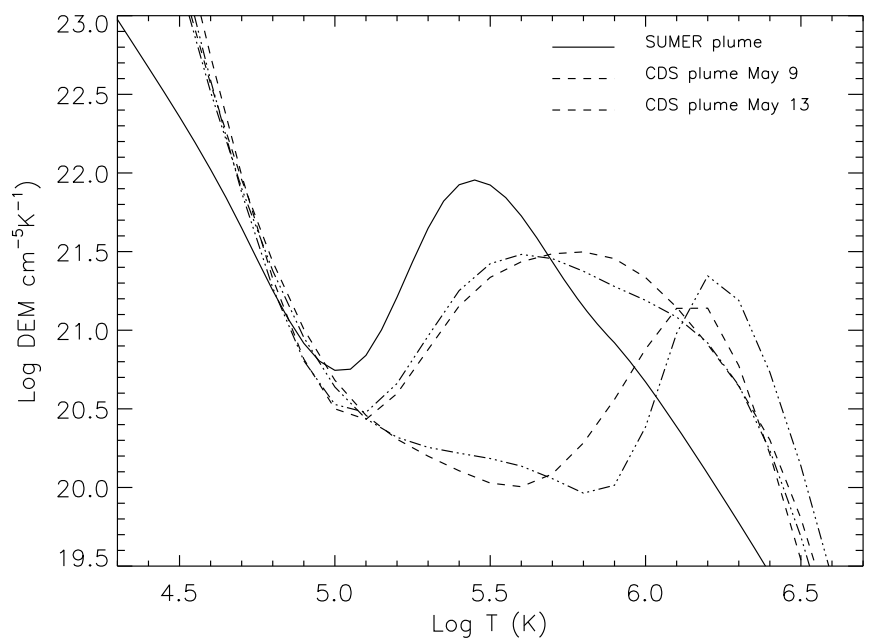

Fig. 6. Comparison of the plume DEM curve with the results of Brosius \& Landi (2005).

Feldman et al. (2009). If we approximate the DEM curves below $\log (T / \mathrm{K})=5.0$ as

$D E M=a \log T+b$

the slope $a$ of the DEM is similar to the values found by Feldman et al. (2009) in coronal hole, quiet Sun, and active region spectra. We note that in Brosius \& Landi (2005) the DEM at lower temperatures (less than $\log (T / \mathrm{K})=5.0$ ) was poorly determined because of the lack of low-temperature lines. Our calculation includes some lines within this temperature range and thus can determine accurately the corresponding thermal structures. At higher temperatures the normalized curves of umbra, penumbra, and plage are all similar but the relative heights of their peaks are different, the umbra DEM peak being higher than both the penumbra and the plage one. The plume peak is the highest of all, although it occurs at lower temperatures.

The plume DEM curve that we measured and the one determined by Brosius \& Landi (2005) are compared in Fig. 6. Brosius \& Landi (2005) measured the DEM of a plume in two different days, and found similar results: their DEM curves were broad and stretched in the entire $5.4 \leq \log (T / \mathrm{K}) \leq 6.0$ temperature range. They also measured the DEM of the quiet Sun in both days, and those curves, with a very narrow peak in the $\log (T / K)=6.1-6.3$ range, are also shown in Fig. 6 for comparison. The plume DEM we measured (solid line in Fig. 6) has a much narrower peak at slightly lower temperatures. One reason for these differences can of course be an intrinsic variability in the sunspot plume thermal structure. Unfortunately, to the best of our knowledge no other DEM measurements of plumes have been made, so this conclusion needs to be confirmed. Another cause of discrepancy might be that Brosius \& Landi (2005) used CDS spectra to determine the curves in Fig. 6. In these spectra, many more lines formed at $\log (T / \mathrm{K})>5.9$ were present, so that the coronal component of the DEM was better constrained than in the present work. The presence of a larger coronal component than in our plume curve might alter the shape of the DEM at temperatures higher than $\log (T / \mathrm{K})=5.8$. In general, we can say that the main signature of sunspot plumes is a greatly enhanced $\mathrm{DEM}$ at transition region temperatures, while at $\log (T / \mathrm{K})<5.0$ the slope of the DEM curve is similar to that of any other region in the Sun, indicating that the thermal structures across this temperature range are fairly similar.
We have to mention that the density and DEM calculations are based on the assumption of ionization equilibrium. In the TR with the strong temperature gradient and presence of significant flows, this assumption is not necessarily valid (Wilhelm et al. 2004). This, as well as the inhomogeneity and variability of the TR plasma, might be important to the determination of electron densities and DEM. However, recent investigations using 3-D model indicated that the assumption of ionization equilibrium might not be too bad in the coronal and TR plasma (Peter et al. 2004, 2006). The calculations in Peter et al. (2006) showed that the ionization and recombination times are at least not (much) longer and often shorter than the typical hydrodynamic timescales. Peter et al. (2006) also found that flows with typical values of $10 \mathrm{~km} \mathrm{~s}^{-1}$ lead to a more shallow temperature gradient and thus help to maintain an ionization equilibrium. Thus, the approximation of ionization equilibrium in our study should not be too bad.

\section{Filling factor}

Although it is well known that the emission of sunspot plumes is greatly enhanced at TR temperatures, the reason for the enhanced intensity remains unknown.

Our measurement reveals a higher intensity in the plume than in the sunspot umbra, penumbra, and the surrounding plage region. The enhanced intensity cannot result from a higher density, since the derived TR densities of the plume are similar to those of the umbra and even much lower than those of the penumbra and plume. It might therefore be an effect of filling factor or temperature, or both.

By using temperature-sensitive line pairs, Doyle et al. (1985) derived the electron temperatures in sunspot plumes. They concluded that the ions are shifted to lower temperatures in the sunspot plume. In our reference spectra, we have no suitable temperature-sensitive line pairs that were observed simultaneously. Thus, this conclusion cannot be examined. However, our DEM analysis clearly reveals that the emitting material of the sunspot plume is concentrated at lower temperatures (higher peak at low $T$ ), which is indicative of a lower plume temperature, compared to that of the surrounding plasma.

With the measurements of line intensities and electron densities, we are able to calculate the filling factor. As mentioned in Dere (2008), the observed intensity of a spectral line emitted by a coronal loop is

$I=\frac{1}{4 \pi} \int G\left(T, N_{\mathrm{e}}\right) N_{\mathrm{e}} N_{\mathrm{H}} \mathrm{d} h$,

where $G(T), N_{\mathrm{e}}, N_{\mathrm{H}}$, and $\mathrm{d} h$ represent the contribution function, electron density, hydrogen density, and the differential of the integration, respectively. Based on the assumption of an isothermal emission feature, we can evaluate the contribution function at its peak temperature $T_{\max }$. The ratio of the hydrogen density relative to the electron density is about 0.8 in a completely ionized plasma of cosmic composition (Landi \& Landini 1997). Usually a loop is not completely filled with emitting plasma, thus a volumetric filling factor should be introduced to account for this. Taking into account these considerations, Eq. (7) can be rewritten as

$I=\frac{0.8}{4 \pi} G\left(T_{\max }, N_{\mathrm{e}}\right) N_{\mathrm{e}}^{2} f L$

where $f$ is the filling factor and $L$ is the length of the integration path. 
Table 3. The details of the filling factor calculations, for the $\mathrm{O} v$ emission of the sunspot plume observed in 2006.

\begin{tabular}{cccccc}
\hline \hline Line $(\AA)$ & $I$ & $\log N_{\mathrm{e}}$ & $G\left(T_{\max }, N_{\mathrm{e}}\right)$ & $L(\mathrm{Mm})$ & $f$ \\
\hline 758.68 & 190.77 & 10.12 & $2.81 \times 10^{-25}$ & 0.5 & 0.096 \\
& & & & 7.0 & 0.007 \\
759.43 & 154.72 & 10.09 & $2.15 \times 10^{-25}$ & 0.5 & 0.120 \\
& & & & 7.0 & 0.009 \\
761.99 & 202.43 & 10.03 & $2.61 \times 10^{-25}$ & 0.5 & 0.160 \\
& & & & 7.0 & 0.012 \\
774.51 & \multirow{2}{*}{44.19} & \multirow{2}{*}{10.03} & $5.54 \times 10^{-26}$ & 0.5 & 0.170 \\
& & & & 7.0 & 0.012 \\
\hline
\end{tabular}

The units of $I, N_{\mathrm{e}}$ and $G\left(T_{\max }, N_{\mathrm{e}}\right)$ are erg cm $\mathrm{cm}^{-2} \mathrm{~s}^{-1} \mathrm{sr}^{-1}, \mathrm{~cm}^{-3}$ and erg cm $\mathrm{cm}^{-3} \mathrm{~s}^{-1} \mathrm{sr}^{-1}$, respectively.

Table 4. The ratios of the filling factors in the four regions, for the $\mathrm{O} \mathrm{V}$ emission in the 2006 data set.

\begin{tabular}{rc}
\hline \hline Line $(\AA)$ & Ratio (umbra : penumbra : plage : plume) \\
\hline 758.68 & $70.8: 1.0: 6.3: 846.2$ \\
759.43 & $211.8: 1.0: 9.6: 2058.8$ \\
761.99 & $114.3: 1.0: 5.9: 1507.9$ \\
774.51 & $69.3: 1.0: 2.4: 666.7$ \\
\hline
\end{tabular}

We used four $\mathrm{O} v$ lines to determine the filling factor of the sunspot plume. The O v $774.51 \AA$ line is density independent, and the other three lines have been used to measure the electron densities. Table 3 lists the calculation details from Eq. (8). Here the intensity refers to that averaged over the plume part along the slit and was converted into energy units by using the procedure of radiometry.pro in SSW (SolarSoft). The contribution function at its peak temperature $T_{\max }$ is density dependent and was calculated at the corresponding density from the CHIANTI database. The factor $1 / 4 \pi$ is included in the $G\left(T_{\max }, N_{\mathrm{e}}\right)$ calculation.

It is difficult to estimate $L$. However, we may be able to provide an upper and a lower limit to this parameter. In the case of ionization equilibrium, the major part of the $\mathrm{O} V$ emission comes from plasmas in the range of $5.2 \leq \log (T / \mathrm{K}) \leq 5.5$. By using a new model of quiet-Sun chromosphere and transition region, Avrett and Loeser (2008) determined the distribution of temperature with height. Using their results, the span of the plasma in the range of $5.2 \leq \log (T / \mathrm{K}) \leq 5.5$ is around $500 \mathrm{~km}$. We assumed this value to be the lower limit to $L$. Since plumes might be related to the high-arching loops observed in ARs (active regions), we may use the extension of the $\mathrm{O} V$ emission in cross-limb AR loop observations as the upper limit to $L$. An example of this type of observation can be found in Brekke et al. (1997), where the AR loop width is about $10^{\prime \prime}$. Thus, we adopted a value of $7 \mathrm{Mm}$ as the upper limit.

The derived filling factors turn out to be relatively large, which might explain the very strong TR emission of sunspot plumes. To compare the filling factor of the plume with those of other features, we can assume that the integration paths in various regions are the same, although this assumption might not be realistic due to a possible difference in their thermal and magnetic structures. Again we obtained the average intensities of the four regions, and calculated $G\left(T_{\max }\right)$ at corresponding densities by using the CHIANTI database. The ratios of the filling factors in the four regions are listed in Table 4.

The results show that the filling factor of the sunspot plume at TR temperatures is between one and three orders of magnitude higher than for the surrounding regions. The difference is so large that it seems safe to conclude that the strong TR emission of sunspot plumes is mainly the result of a large filling factor. However, we should not exclude the possibility that other effects, e.g., the region emitting TR lines being much thicker in sunspot plumes, might also play a role in producing the strong emission.

\section{Peculiar lines in the sunspot}

As mentioned in our earlier paper (Curdt et al. 2000), more than 100 "peculiar" lines including several $\mathrm{H}_{2}$ lines are present in the sunspot reference spectra obtained on March 18, 1999. These lines are also found in the sunspot atlas presented by Curdt et al. (2001). Most of these lines are upper-TR lines with a temperature range of $5.3 \leq(\log (T / \mathrm{K}) \leq 6.0$, corresponding to 4 to 8 -fold ionized species. Here we confirm that these "peculiar" lines are also present in all of the 5 analyzed data sets. These lines are weak or not observed in either the quiet Sun and or corona. Some of them may be present in streamer spectra.

Many of these "peculiar" lines correspond to forbidden transitions. Thus, we may speculate that an extremely low density might be responsible for their formation. However, as we mentioned previously, the umbra and plume densities derived here are similar to, or slightly higher than the density of the normal quiet Sun. This means that the sunspot plasma density is not extremely low and the above explanation is questionable.

Through a visual inspection of the spectra analyzed here and several other sunspot reference spectra, we found that the "peculiar" lines seem more likely to be associated with plumes, rather than the umbrae. One possible reason why these lines are present in the umbra (Curdt et al. 2001) is that part of the sunspot plumes are just located above the umbra and the sample of the sunspot emission might be a mixture of the plume and umbra emissions. Since the plume emission is much stronger than the umbra emission at TR temperatures, the average sunspot spectrum in Curdt et al. (2001) is dominated by the plume spectrum.

The reason why these "peculiar" lines are so distinctive in the sunspot plume is probably a combination of two effects. First, the plume emission is strongest at upper-TR temperatures. Second, the continuum emission of the plume is much weaker than that of the plage.

We list these "peculiar" lines in Table 6. As an example, in Fig. 7 we show part of the spectra observed in the plage, penumbra, umbra, and plume for the 2006 data set. We see that several forbidden lines (e.g., Mg VII 854.75 Ne VII $895.19 \AA$ ) become very prominent in the plume spectrum. Here, we also see in the umbra spectrum the enhanced forbidden lines, which are not so enhanced as in the plume and might be due to contamination from the plume emission at the formation temperatures of these lines. However, the strongly reduced continuum emission of the umbra may also cause the forbidden lines to be distinctive.

The $\mathrm{H}_{2}$ emission is extremely weak in the quiet Sun (Sandlin et al. 1986). It was also observed in flares (Bartoe et al. 1979) and microflares in the active region plage (Innes 2008). In sunspot regions, the $\mathrm{H}_{2}$ emission is distinctive and relatively strong (Jordan et al. 1978; Schühle et al. 1999; Curdt et al. 2001). Several lines of the $\mathrm{H}_{2}$ Werner bands are within the SUMER spectral range. These lines are believed to be excited by resonance fluorescence through the strong O VI $1031.9 \AA$ line. In our data, the emission of these lines is clearly detected in the umbra and plume regions, which might be related to the reduced opacity above the sunspot. The O VI 1031.9 $\AA$ emission is able to reach the chromosphere and excite the $\mathrm{H}_{2}$ lines if the opacity is low. 


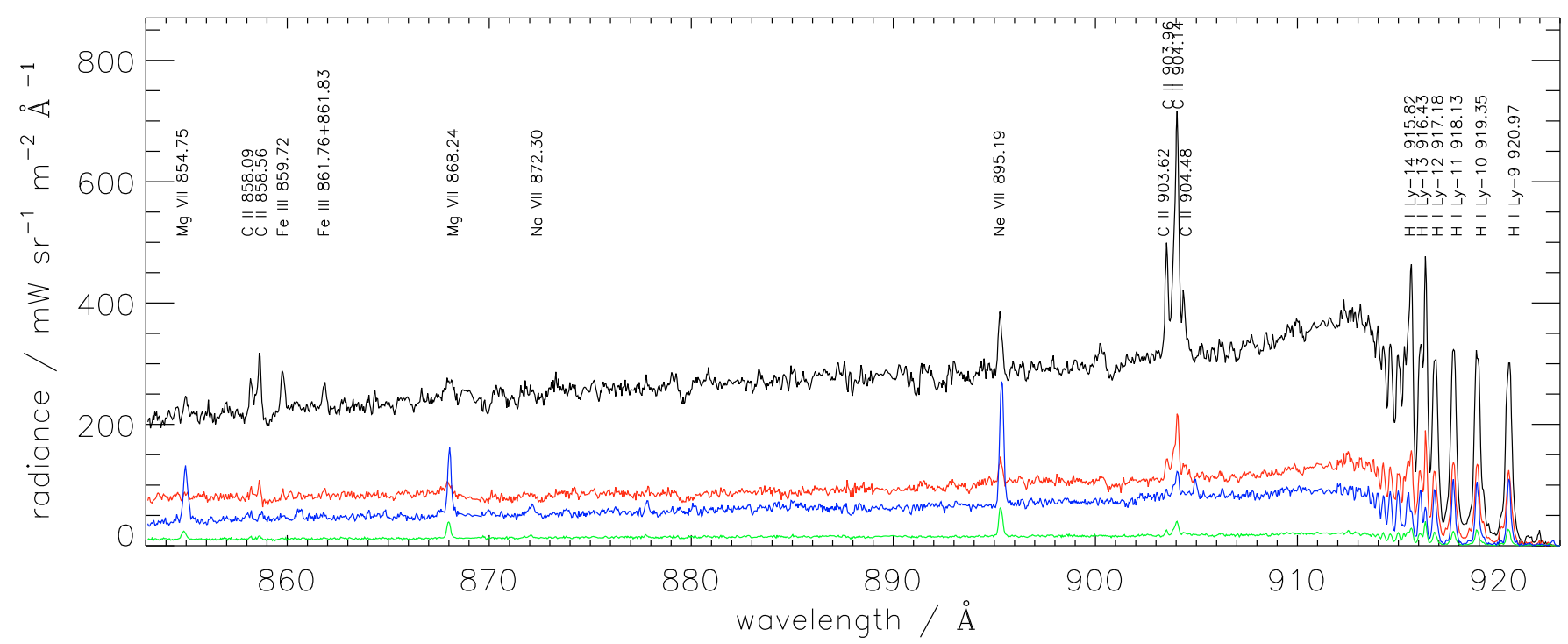

Fig. 7. Part of the spectra observed in the plage, penumbra, umbra and plume for the 2006 data set. The black, red, green and blue lines correspond to the plage, penumbra, umbra and plume spectra.

\section{Summary}

By analyzing sunspot reference spectra taken by the SUMER instrument, we have shown that the TR above sunspots has some distinctive properties compared to the surrounding plage regions.

We have found that the hydrogen Lyman line profiles are not reversed in sunspots at different locations (heliocentric angle up to $49^{\circ}$ ) on the solar disk. The Lyman lines are also not reversed in sunspot plumes. In the plage region, the lower Lyman line profiles are obviously reversed, a phenomenon also found in the normal quiet Sun. Line-pair diagnostics yields an electron density of $\log \left(N_{\mathrm{e}} / \mathrm{cm}^{-3}\right) \approx 10.0$ for the umbra and the plume, and $\approx 11.0$ for the penumbra and plage, at TR temperatures. To explain these results, we suggest that the TR above sunspots is higher and probably more extended, and the opacity above sunspots is much lower than in the TR above plage regions.

We also completed a DEM analysis for the sunspot observed in 2006. To the best of our knowledge, this is the first time that SUMER spectra have been used for the DEM diagnostics of a sunspot. The DEM curve of the plume is obviously different from those of other regions. It peaks at a lower temperature of around $\log (T / \mathrm{K})=5.45$, which exceeds the DEM of other regions by one to two orders of magnitude at these temperatures. At $\log (T / K)<5.0$, the slope of the DEM curve is similar in the four regions, indicating that the thermal structure in this temperature range is fairly similar everywhere in and around the sunspots.

The reason why the plume emission is so strong at upper-TR temperatures has been investigated for the first time. Our calculations seem to indicate that the enhanced TR emission of the sunspot plume is very likely to be the result of a large filling factor.

More than 100 lines that are rather weak or not observed anywhere else on the Sun, are well observed by SUMER in the sunspot, especially in the sunspot plume. We propose that it is the combination of strongly enhanced emission at TR temperatures and reduced continuum cause these normally weak lines to be clearly distinctive in the spectra of sunspot plumes.

Acknowledgements. The SUMER project is financially supported by DLR, CNES, NASA, and the ESA PRODEX Programme (Swiss contribution).
SUMER is an instrument onboard $\mathrm{SOHO}$, a mission operated by ESA and NASA. We thank Dr. D. E. Innes and Dr. H. Peter for the helpful comments. Hui Tian is supported by the IMPRS graduate school run jointly by the Max Planck Society and the Universities of Göttingen and Braunschweig. The work of Hui Tian's group at PKU is supported by the National Natural Science Foundation of China (NSFC) under contract 40874090. Enrico Landi acknowledges support from the NNG06EA14I, NNH06CD24C NASA grants.

\section{References}

Avrett, E. H., \& Loeser, R. 2008, ApJS, 175, 229

Bartoe, J.-D. F., Brueckner, G. E., Purcell, J. D., et al. 1977, Appl. Opt., 16, 879 Bartoe, J.-D. F., Brueckner, G. E., Nicolas, K. R., et al. 1979, MNRAS, 187, 463 Brekke, P., Kjeldseth-Moe, O., \& Harrison, R. A. 1997, Sol. Phys., 175, 511

Brooks, D. H., Fischbacher, G. A., Fludra, A., et al. 2000, A\&A, 357, 697 Brosius, J. W. 2005, ApJ, 622, 1216

Brosius, J. W., \& Landi, E. 2005, ApJ, 632, 1196

Brynildsen, N., Maltby, P., Brekke, P., Haugan, S. V. H., \& Kjeldseth-Moe, O. 1999a, Sol. Phys., 186, 141

Brynildsen, N., Maltby, P., Leifsen, T., Kjeldseth-Moe, O., \& Wilhelm, K. 1999b, Sol. Phys., 191, 129

Brynildsen, N., Maltby, P., Fredvik, T., et al. 2001, Sol. Phys., 198, 89

Brueckner, G. E., \& Bartoe, J.-D. F. 1983, ApJ, 272, 329

Brueckner, G. E., Bartoe, J.-D. F., \& VanHoosier, M. E. 1977, in Proc. OSO-8 Workshop, ed. E. Hansen, \& S. Schaffner (Boulder: Univ. Colorado Press), 380

Brueckner, G. E., Bartoe, J.-D. F., Cook, J. W., Dere, K. P., \& Socker, D. G. 1986, Adv. Space Res., 6, 263

Curdt, W., Dwivedi, B. N, \& Feldman, U. 2000, A\&A, 21, 397

Curdt, W., Brekke, P., Feldman, U., et al. 2001, A\&A 375, 591

Curdt, W., Landi, E., \& Feldman, U. 2004, A\&A, 427, 1045

Curdt, W., Tian, H., Teriaca, L., Schühle, U., \& Lemaire, P. 2008, A\&A, 492, L9

Dammasch, I. E., Curdt, W., Dwivedi, B. N., et al. 2008, Ann. Geophys., 26, 2955

Dere, K. P. 2008, A\&A, 491, 561

Dere, K. P., Landi, E., Mason, H. E., et al. 1997, A\&ASS, 125, 149

Dowdy, J. F., Jr., Rabin, D., \& Moore, R. L. 1986, Sol. Phys., 105, 35

Doyle, J. G., \& Madjarska, M. S. 2003, A\&A, 407, L29

Doyle, J. G., Raymond, J. C., Noyes, R. W., et al. 1985, ApJ, 297, 816

Dupree, A. K. 1972, ApJ, 178, 527

Ekberg, J. O., \& Feldman, U. 2003, ApJS, 148, 567

Esser, R., Lie-Svendsen, Ø, Janse, A. M., et al. 2005, ApJ, 629, L61

Fang, C., Feautrier, N., \& Hénoux, J.-C. 1995, A\&A, 297, 854

Feldman, U. 1983, ApJ, 275, 367

Feldman, U. 1987, ApJ, 320, 426

Feldman, U., \& Laming, J. M. 2000, Phys. Scr., 61, 222

Feldman, U., Behring, W. E., Curdt, W., et al. 1997, ApJS, 113, 195

Feldman, U., Dammasch, I. E., \& Landi, E. 2009, ApJ, 693, 1474

Fontenla, J. M., Reichmann, E. J., \& Tandberg-Hanssen, E. 1988, ApJ, 329, 464 
Foukal, P. V. 1976, ApJ, 210, 575

Foukal, P. V., Huber, M. C. E., Noyes, R. W., et al. 1974, ApJ, 193, L143

Gabriel, A. H. 1976, Philos. Trans. R. Soc. London A, 281, 575

Grevesse, N., \& Sauval, A. J. 1998, Space Sci. Rev., 85, 161

Griffiths, N. W., Fisher, G. H., Woods, D. T., et al. 1999, ApJ, 512,992

Gunár, S., Heinzel, P., Anzer, U., et al. 2008, A\&A, 490, 307

Guo, Y., Ding, M. D., Jin, M., et al. 2009, ApJ, 696, 1526

Harrison, R. A., \& Thompson, A. M. 1992, Intensity integral inversion techniques: a study in preparation for the SOHO mission, RAL-91-092, Rutherford Appleton Laboratory

Harrison, R. A., Sawyer, E. C., Carter, M. K., et al. 1995, Sol. Phys.,1995, 162, 233

Heinzel, P., Anzer, U., \& Gunár, S. 2005, A\&A, 442, 331

Innes, D. E. 2008, A\&A, 481, L41

Jordan, C., Brueckner, G. E., Bartoe, J.-D. F., et al. 1978, ApJ, 226, 687

Kjeldseth-Moe, O., Brynildsen, N., Brekke, P., et al. 1988, ApJ, 334, 1066

Landi, E., \& Landini, M. 1997, A\&A, 327, 1230

Landi, E., Feldman, U., \& Dere, K. P. 2002, ApJS, 139, 281

Landi, E., Del Zanna, G., Young, P. R., et al. 2006, ApJS, 162, 261

Lemaire, P., Wilhelm, K., Curdt, W., et al. 1997, Sol. Phys., 170, 105

Mariska, J. T. 1988, ApJ, 334, 489

Mariska, J. T. 1992, The Solar Transition Region (Cambridge: Cambridge Univ. Press)

Marsch, E., Tu, C.-Y., Heinzel, P., Wilhelm, K., \& Curdt, W. 1999, A\&A, 347, 676

Marsch, E., Tu, C.-Y., \& Wilhelm, K. 2000, A\&A, 359, 381

Marsch, E., Wiegelmann, T., \& Xia L.-D. 2004, A\&A, 428, 629

Marsch, E., Zhou, G.-Q., He, J.-S., et al. 2006, A\&A, 457, 699

McClymont, A. N., \& Craig, I. J. D. 1987, ApJ, 312, 402

Mazzotta, P., Mazzitelli, G., Colafrancesco, S., et al. 1998, A\&AS, 133, 197

Nicolas, K. R., Kjeldseth-Moe, O., Bartoe, J.-D. F., et al. 1982, Sol. Phys., 81, 253
Noyes, R. W., Raymond, J. C., Doyle, J. G., et al. 1985, ApJ, 297, 805 Peter, H. 2001, A\&A, 374,1108

Peter, H., Gudiksen, B., \& Nordlund, Å. 2004, ApJ, 617, L85

Peter, H., Gudiksen, B., \& Nordlund, Å. 2006, ApJ, 638, 1086

Phillips, K. J. H., Feldman, U., \& Landi, E. 2008, Ultraviolet and X-ray spectroscopy of the solar atmosphere, Cambridge Astrophysics Series 44 (Cambridge University Press)

Ralchenko, Yu., Kramida, A. E., Reader, J., \& NIST ASD Team (2008), NIST Atomic Spectra Database (version 3.1.5). http://physics.nist.gov/ asd3. National Institute of Standards and Technology, Gaithersburg, MD

Sandlin, G. D., Bartoe, J.-D. F., \& Brueckner, G. E. 1986, ApJS, 61, 801

Schmieder, B., Gunár, S., Heinzel, P., et al. 2007, Sol. Phys. 241, 53

Schühle, U., Brown, C. M., Curdt, W., et al. 1999, in Proc. SOHO 8, ESA SP446,617

Solanki, S. K. 2003, Astron. Astrophys. Rev., 11, 153

Spadaro, D., Antiochos, S. K., \& Mariska, J. T. 1991, ApJ 382, 338

Tian, H., Marsch, E., Tu, C.-Y., Xia, L.-D., \& He, J.-S. 2008a, A\&A, 482, 267

Tian, H., Xia, L.-D., He, J.-S., Tan, B., \& Yao, S. 2008b, Chin. J. Astron. Astrophys., 8, 6, 732

Tian, H., Curdt, W., Marsch, E., Schühle, U. 2009, A\&A, 504, 239

Teriaca, L., Curdt, W., \& Solanki, S. K. 2008, A\&A, 491, L5

Tu, C.-Y., Zhou, C., Marsch, E., et al. 2005a, Science, 308, 519

Tu, C.-Y., Zhou, C., Marsch, E., et al. 2005b, ApJ, 624, L133

Vial, J. C., Ebadi, H., \& Ajabshirizadeh, A. 2007, Sol. Phys. 246, 327

Warren, H. P., Mariska, J. T., \& Wilhelm, K. 1998, ApJS 119, 105

Wilhelm, K., Curdt, W., Marsch, E., et al. 1995, Sol. Phys. 162, 189

Wilhelm, K., Dviwedi, B. N., Marsch, E., et al. 2004, Space Sci. Rev., 111, 415

Wilhelm, K., Marsch, E., Dviwedi, B. N., et al. 2007, Space Sci. Rev., 133, 103

Xia, L.-D. 2003, Ph.D. Thesis (Göttingen: Georg-August-Univ.)

Xia, L.-D., Marsch, E., \& Wilhelm, K. 2004, A\&A, 424, 1025

Xu, Z., Fang, C., \& Gan, W.-Q. 2005, Chin. J. Astron. Astrophys., 5, 519 
H. Tian et al.: Solar transition region above sunspots, Online Material $p 1$

Table 5. List of lines used for DEM diagnostics. The values of intensities (in the unit of photon $\mathrm{sr}^{-1} \mathrm{~s}^{-1} \operatorname{arcsec}^{-2}$ ) of the four regions are also shown.

\begin{tabular}{|c|c|c|c|c|c|c|c|}
\hline Ion & $\lambda_{\mathrm{obs}}$ & $\begin{array}{l}\text { Transition } \\
\end{array}$ & $\log \left(T_{f} / \mathrm{K}\right)$ & Umbra & Penumbra & Plage & Plume \\
\hline C II & 903.590 & $2 \mathrm{~s}^{2} 2 \mathrm{p}^{2} \mathrm{P}_{1 / 2}-2 \mathrm{~s} 2 \mathrm{p}^{2}{ }^{2} \mathrm{P}_{3 / 2}$ & 4.37 & $1.924 \mathrm{e}+00$ & $1.018 \mathrm{e}+01$ & $4.125 e+01$ & $4.625 \mathrm{e}+00$ \\
\hline $\mathrm{C}$ II & 903.990 & $2 \mathrm{~s}^{2} 2 \mathrm{p}^{2} \mathrm{P}_{1 / 2}-2 \mathrm{~s} 2 \mathrm{p}^{2}{ }^{2} \mathrm{P}_{1 / 2}$ & & $1.826 \mathrm{e}+00$ & $1.124 \mathrm{e}+01$ & $2.636 e+01$ & $3.832 \mathrm{e}+00$ \\
\hline $\mathrm{C}$ II & 904.140 & $2 \mathrm{~s}^{2} 2 \mathrm{p}^{2} \mathrm{P}_{3 / 2}-2 \mathrm{~s} 2 \mathrm{p}^{2}{ }^{2} \mathrm{P}_{3 / 2}$ & & $5.342 \mathrm{e}+00$ & $2.528 \mathrm{e}+01$ & $8.981 \mathrm{e}+01$ & $1.010 \mathrm{e}+01$ \\
\hline $\mathrm{C}$ II & 904.460 & $2 \mathrm{~s}^{2} 2 \mathrm{p}^{2} \mathrm{P}_{3 / 2}-2 \mathrm{~s} 2 \mathrm{p}^{2}{ }^{2} \mathrm{P}_{1 / 2}$ & & $1.106 e+00$ & $9.160 \mathrm{e}+00$ & $2.566 \mathrm{e}+01$ & $3.981 \mathrm{e}+00$ \\
\hline $\mathrm{C}$ II & 1036.340 & $2 \mathrm{~s}^{2} 2 \mathrm{p}^{2} \mathrm{P}_{1 / 2}-2 \mathrm{~s} 2 \mathrm{p}^{2}{ }^{2} \mathrm{~S}_{1 / 2}$ & & $2.527 \mathrm{e}+01$ & $9.625 e+01$ & $4.045 e+02$ & $3.452 \mathrm{e}+01$ \\
\hline $\mathrm{C}$ II & 1037.000 & $2 \mathrm{~s}^{2} 2 \mathrm{p}^{2} \mathrm{P}_{3 / 2}-2 \mathrm{~s} 2 \mathrm{p}^{2}{ }^{2} \mathrm{~S}_{1 / 2}$ & & $3.181 \mathrm{e}+01$ & $1.429 \mathrm{e}+02$ & $4.705 e+02$ & $4.227 \mathrm{e}+01$ \\
\hline $\mathrm{C}$ II & 1334.530 & $2 \mathrm{~s}^{2} 2 \mathrm{p}^{2} \mathrm{P}_{1 / 2}-2 \mathrm{~s} 2 \mathrm{p}^{2}{ }^{2} \mathrm{D}_{3 / 2}$ & & $7.287 \mathrm{e}+02$ & $8.435 \mathrm{e}+03$ & $2.665 e+04$ & $1.375 \mathrm{e}+03$ \\
\hline $\mathrm{C}_{\text {II }}$ & 1335.710 & $2 \mathrm{~s}^{2} 2 \mathrm{p}^{2} \mathrm{P}_{3 / 2}-2 \mathrm{~s} 2 \mathrm{p}^{2}{ }^{2} \mathrm{D}_{5 / 2}$ & & $1.187 \mathrm{e}+03$ & $1.161 \mathrm{e}+04$ & $3.852 \mathrm{e}+04$ & $2.237 \mathrm{e}+03$ \\
\hline C III & 977.030 & $2 \mathrm{~s}^{2}{ }^{1} \mathrm{~S}_{0}-2 \mathrm{~s}^{2 \mathrm{p}{ }^{1}} \mathrm{P}_{1}$ & 4.84 & $5.350 \mathrm{e}+02$ & $2.381 \mathrm{e}+03$ & $9.698 \mathrm{e}+03$ & $6.622 \mathrm{e}+02$ \\
\hline C III & 1174.880 & $2 \mathrm{~s}^{2 \mathrm{p}{ }^{3}} \mathrm{P}_{1}-2^{\mathrm{p} 2}{ }^{3} \mathrm{P}_{2}$ & & $3.734 \mathrm{e}+01$ & $1.693 \mathrm{e}+02$ & $9.485 e+02$ & $4.486 \mathrm{e}+01$ \\
\hline C III & 1175.240 & $2 \mathrm{~s}^{2 \mathrm{p} 3} \mathrm{P}_{0}-2^{\mathrm{p} 2}{ }^{3} \mathrm{P}_{1}$ & & $3.090 \mathrm{e}+01$ & $1.371 \mathrm{e}+02$ & $8.754 \mathrm{e}+02$ & $3.823 \mathrm{e}+01$ \\
\hline C III & 1175.980 & 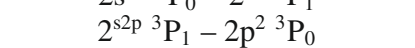 & & $2.954 \mathrm{e}+01$ & $1.301 \mathrm{e}+02$ & $8.114 \mathrm{e}+02$ & $3.805 \mathrm{e}+01$ \\
\hline C III & 1176.370 & $2 \mathrm{~s}^{2 \mathrm{p}{ }^{3}} \mathrm{P}_{2}-2^{\mathrm{p}^{2}{ }^{3}} \mathrm{P}_{1}$ & & $3.493 \mathrm{e}+01$ & $1.690 \mathrm{e}+02$ & $8.597 \mathrm{e}+02$ & $4.494 \mathrm{e}+01$ \\
\hline C III & 690.530 & $2 \mathrm{~s} 2 \mathrm{p}{ }^{1} \mathrm{P}_{1}-2 \mathrm{p}^{2}{ }^{1} \mathrm{~S}_{0}$ & & $8.225 \mathrm{e}-01$ & $2.587 \mathrm{e}+00$ & $9.027 \mathrm{e}+00$ & $1.404 \mathrm{e}+00$ \\
\hline N II & 1083.990 & $2^{\mathrm{s} 2} 2^{\mathrm{p}^{2}{ }^{3}} \mathrm{P}_{0}-2 \mathrm{~s}^{2 \mathrm{p} 3}{ }^{3} \mathrm{D}_{1}$ & 4.43 & $1.064 \mathrm{e}+01$ & $5.329 \mathrm{e}+01$ & $1.531 \mathrm{e}+02$ & $1.347 \mathrm{e}+01$ \\
\hline N II & 1085.540 & $2^{\mathrm{s} 2} 2^{\mathrm{p} 2}{ }^{3} \mathrm{P}_{1}-2^{\mathrm{s} 2 \mathrm{p} 3{ }^{3}} \mathrm{D}_{1,2}$ & & $9.890 \mathrm{e}+00$ & $6.396 \mathrm{e}+01$ & $2.255 \mathrm{e}+02$ & $9.610 \mathrm{e}+00$ \\
\hline N II & 1085.710 & $2 \mathrm{~s}^{2} 2^{\mathrm{p} 2}{ }^{3} \mathrm{P}_{1}-2^{\mathrm{s} 2 \mathrm{p} 3}{ }^{3} \mathrm{D}_{3}$ & & $4.385 e+01$ & $1.719 \mathrm{e}+02$ & $5.054 \mathrm{e}+02$ & $5.968 \mathrm{e}+01$ \\
\hline N II & 746.990 & $2 \mathrm{~s}^{2} 2 \mathrm{p}^{2}{ }^{1} \mathrm{D}_{2}-2 \mathrm{~s}^{2} 2 \mathrm{p} 3^{\mathrm{s}}{ }^{1} \mathrm{P}_{1}$ & & $4.308 \mathrm{e}-01$ & $1.559 \mathrm{e}+00$ & $4.913 \mathrm{e}+00$ & $9.306 \mathrm{e}-01$ \\
\hline $\mathrm{N}$ III & 684.990 & $2 \mathrm{~s}^{2} 2 \mathrm{p}^{2} \mathrm{P}_{1 / 2}-2 \mathrm{~s} 2 \mathrm{p}^{2}{ }^{2} \mathrm{P}_{3 / 2}$ & 4.88 & $1.970 \mathrm{e}+00$ & $4.252 \mathrm{e}+00$ & $1.770 \mathrm{e}+01$ & $3.062 \mathrm{e}+00$ \\
\hline N III & 685.500 & $2 s^{2} 2 p^{2} P_{1 / 2}-2 s 2 p^{2}{ }^{2} P_{1 / 2}$ & & $2.547 \mathrm{e}+00$ & $7.454 \mathrm{e}+00$ & $3.300 \mathrm{e}+01$ & $4.621 \mathrm{e}+00$ \\
\hline N III & 685.790 & $2 s^{2} 2 p^{2} P_{3 / 2}-2 s 2 p^{2}{ }^{2} P_{3 / 2}$ & & $7.789 \mathrm{e}+00$ & $2.054 \mathrm{e}+01$ & $8.704 \mathrm{e}+01$ & $1.334 \mathrm{e}+01$ \\
\hline N III & 763.330 & $2 \mathrm{~s}^{2} 2 \mathrm{p}^{2} \mathrm{P}_{1 / 2}-2 \mathrm{~s} 2 \mathrm{p}^{2}{ }^{2} \mathrm{~S}_{1 / 2}$ & & $1.822 \mathrm{e}+00$ & $6.381 \mathrm{e}+00$ & $2.865 \mathrm{e}+01$ & $4.770 \mathrm{e}+00$ \\
\hline N III & 764.360 & $2 \mathrm{~s}^{2} 2 \mathrm{p}^{2} \mathrm{P}_{3 / 2}-2 \mathrm{~s} 2 \mathrm{p}^{2}{ }^{2} \mathrm{~S}_{1 / 2}$ & & $2.803 \mathrm{e}+00$ & $9.623 e+00$ & $4.852 \mathrm{e}+01$ & $7.042 \mathrm{e}+00$ \\
\hline N III & 989.820 & $2 \mathrm{~s}^{2} 2 \mathrm{p}^{2} \mathrm{P}_{1 / 2}-2 \mathrm{~s} 2 \mathrm{p}^{2}{ }^{2} \mathrm{D}_{3 / 2}$ & & $1.345 \mathrm{e}+01$ & $4.559 \mathrm{e}+01$ & $1.764 \mathrm{e}+02$ & $2.733 \mathrm{e}+01$ \\
\hline N IV & 765.150 & $2 \mathrm{~s}^{2}{ }^{1} \mathrm{~S}_{0}-2 \mathrm{~s} 2 \mathrm{p}{ }^{1} \mathrm{P}_{1}$ & 5.15 & $5.743 e+01$ & $1.269 \mathrm{e}+02$ & $6.247 \mathrm{e}+02$ & $3.949 \mathrm{e}+02$ \\
\hline $\mathrm{N} \mathrm{V}$ & 1238.820 & $2 s 2^{S 1 / 2}-2 p^{2} P_{3 / 2}$ & 5.26 & $1.858 \mathrm{e}+02$ & $5.114 \mathrm{e}+02$ & $2.296 \mathrm{e}+03$ & $1.093 \mathrm{e}+03$ \\
\hline $\mathrm{NV}$ & 1242.800 & $2 \mathrm{~s} 2^{S 1 / 2}-2 \mathrm{p}^{2} \mathrm{P}_{1 / 2}$ & & $8.740 \mathrm{e}+01$ & $2.233 \mathrm{e}+02$ & $1.046 \mathrm{e}+03$ & $5.191 \mathrm{e}+02$ \\
\hline O II & 796.660 & $2 \mathrm{~s}^{2} 2 \mathrm{p}^{32} \mathrm{P}_{1 / 2,3 / 2}-2 \mathrm{~s} 2 \mathrm{p}^{4}{ }^{2} \mathrm{D}_{3 / 2,5 / 2}$ & 4.45 & $1.531 \mathrm{e}+00$ & $3.672 \mathrm{e}+00$ & $2.705 \mathrm{e}+01$ & $5.133 \mathrm{e}+00$ \\
\hline O II & 832.750 & $2 \mathrm{~s}^{2} 2 \mathrm{p}^{3} 4^{S 3 / 2}-2 \mathrm{~s} 2 \mathrm{p}^{4}{ }^{4} \mathrm{P}_{1 / 2}$ & & $3.860 \mathrm{e}+00$ & $1.599 \mathrm{e}+01$ & $5.618 \mathrm{e}+01$ & $8.631 \mathrm{e}+00$ \\
\hline O II & 833.320 & $2 s^{2} 2 p^{3} 4^{S 3 / 2}-2 s 2 p^{4}{ }^{4} P_{3 / 2}$ & & $1.024 \mathrm{e}+01$ & $4.465 e+01$ & $1.791 \mathrm{e}+02$ & $2.034 \mathrm{e}+01$ \\
\hline O II & 834.450 & $2 s^{2} 2 p^{3} 4^{S 3 / 2}-2 s 2 p^{4}{ }^{4} P_{5 / 2}$ & & $1.349 \mathrm{e}+01$ & $6.033 \mathrm{e}+01$ & $2.460 \mathrm{e}+02$ & $2.092 \mathrm{e}+01$ \\
\hline O III & 702.330 & $2^{\mathrm{s} 2} 2^{\mathrm{p} 2} 3^{\mathrm{P} 0}-2^{\mathrm{s} 2 \mathrm{p} 3}{ }^{3} \mathrm{P}_{1}$ & 4.93 & $6.518 \mathrm{e}+00$ & $1.267 \mathrm{e}+01$ & $4.592 \mathrm{e}+01$ & $1.746 \mathrm{e}+01$ \\
\hline O III & 703.870 & $2^{\mathrm{s} 2} 2^{\mathrm{p} 2} 3^{\mathrm{P} 1}-2^{\mathrm{s} 2 \mathrm{p} 3}{ }^{3} \mathrm{P}_{1,2}$ & & $2.763 e+01$ & $5.699 \mathrm{e}+01$ & $1.870 \mathrm{e}+02$ & $7.773 \mathrm{e}+01$ \\
\hline O III & 832.940 & $2^{\mathrm{s} 2} 2^{\mathrm{p} 2} 3^{\mathrm{P} 0}-2 \mathrm{~s} 2 \mathrm{p}^{3}{ }^{3} \mathrm{D}_{1}$ & & $7.761 \mathrm{e}+00$ & $3.194 \mathrm{e}+01$ & $1.661 \mathrm{e}+02$ & $4.104 \mathrm{e}+01$ \\
\hline O III & 833.740 & $2 s^{2} 2 p^{2} 3^{\mathrm{P} 1}-2 \mathrm{~s} 2 \mathrm{p}^{3}{ }^{3} \mathrm{D}_{1,2}$ & & $1.880 \mathrm{e}+01$ & $8.064 \mathrm{e}+01$ & $3.955 \mathrm{e}+02$ & $1.272 \mathrm{e}+02$ \\
\hline O III & 835.090 & $2 s^{2} 2 p^{2} 3^{\mathrm{P} 2}-2 \mathrm{~s} 2 \mathrm{p}^{3}{ }^{3} \mathrm{D}_{1,2}$ & & $6.567 \mathrm{e}+00$ & $2.925 \mathrm{e}+01$ & $8.284 \mathrm{e}+01$ & $2.850 \mathrm{e}+01$ \\
\hline O III & 835.280 & $2 \mathrm{~s}^{2} 2 \mathrm{p}^{2} 3^{\mathrm{P} 2}-2 \mathrm{~s} 2 \mathrm{p}^{3}{ }^{3} \mathrm{D}_{3}$ & & $2.757 e+01$ & $1.123 \mathrm{e}+02$ & $6.207 e+02$ & $1.840 \mathrm{e}+02$ \\
\hline O IV & 787.720 & $2 \mathrm{~s}^{2} 2 \mathrm{p}^{2} \mathrm{P}_{1 / 2}-2 \mathrm{~s} 2 \mathrm{p}^{2}{ }^{2} \mathrm{D}_{3 / 2}$ & 5.18 & $6.234 \mathrm{e}+01$ & $8.764 \mathrm{e}+01$ & $4.506 \mathrm{e}+02$ & $9.172 \mathrm{e}+02$ \\
\hline OIV & 790.190 & $2 \mathrm{~s}^{2} 2 \mathrm{p}^{2} \mathrm{P}_{3 / 2}-2 \mathrm{~s} 2 \mathrm{p}^{2}{ }^{2} \mathrm{D}_{3 / 2,5 / 2}$ & & $1.291 \mathrm{e}+02$ & $2.000 \mathrm{e}+02$ & $1.032 \mathrm{e}+03$ & $1.990 \mathrm{e}+03$ \\
\hline OIV & 1397.220 & $2 \mathrm{~s}^{2} 2 \mathrm{p}^{2} \mathrm{P}_{1 / 2}-2 \mathrm{~s} 2 \mathrm{p}^{2}{ }^{4} \mathrm{P}_{3 / 2}$ & & $4.660 \mathrm{e}+00$ & $1.850 \mathrm{e}+01$ & $2.898 \mathrm{e}+01$ & $1.978 \mathrm{e}+01$ \\
\hline O IV & 1399.770 & $2 \mathrm{~s}^{2} 2 \mathrm{p}^{2} \mathrm{P}_{1 / 2}-2 \mathrm{~s} 2 \mathrm{p}^{2}{ }^{4} \mathrm{P}_{1 / 2}$ & & $1.378 \mathrm{e}+01$ & $6.094 \mathrm{e}+01$ & $1.703 e+02$ & $8.864 \mathrm{e}+01$ \\
\hline OIV & 1401.160 & $2 \mathrm{~s}^{2} 2 \mathrm{p}^{2} \mathrm{P}_{3 / 2}-2 \mathrm{~s} 2 \mathrm{p}^{2}{ }^{4} \mathrm{P}_{5 / 2}$ & & $6.494 e+01$ & $1.915 \mathrm{e}+02$ & $5.799 \mathrm{e}+02$ & $4.078 \mathrm{e}+02$ \\
\hline OIV & 1407.390 & $2 \mathrm{~s}^{2} 2 \mathrm{p}^{2} \mathrm{P}_{3 / 2}-2 \mathrm{~s} 2 \mathrm{p}^{2}{ }^{4} \mathrm{P}_{1 / 2}$ & & $1.629 \mathrm{e}+01$ & $5.708 \mathrm{e}+01$ & $2.056 e+02$ & $9.552 \mathrm{e}+01$ \\
\hline $\mathrm{OV}$ & 758.680 & $2 \mathrm{~s} 2 \mathrm{p}{ }^{3} \mathrm{P}_{1}-2 \mathrm{p}^{2}{ }^{3} \mathrm{P}_{2}$ & 5.37 & $1.126 \mathrm{e}+01$ & $1.565 \mathrm{e}+01$ & $9.466 e+01$ & $1.829 \mathrm{e}+02$ \\
\hline $\mathrm{OV}$ & 760.210 & $2 \mathrm{~s} 2 \mathrm{p}^{3} \mathrm{P}_{1}-2 \mathrm{p}^{2}{ }^{3} \mathrm{P}_{1}$ & & $6.276 \mathrm{e}+00$ & $8.414 \mathrm{e}+00$ & $5.152 \mathrm{e}+01$ & $1.048 \mathrm{e}+02$ \\
\hline $\mathrm{OV}$ & 760.430 & $2 s 2{ }^{3} P_{2}-2 p^{2}{ }^{3} P_{2}$ & & $3.441 \mathrm{e}+01$ & $3.935 \mathrm{e}+01$ & $2.673 e+02$ & $5.590 \mathrm{e}+02$ \\
\hline $\mathrm{Ov}$ & 761.130 & $2 \mathrm{~s} 2 \mathrm{p}^{3} \mathrm{P}_{1}-2 \mathrm{p}^{2}{ }^{3} \mathrm{P}_{0}$ & & $1.958 \mathrm{e}+00$ & $8.264 \mathrm{e}+00$ & $4.919 \mathrm{e}+01$ & $3.561 \mathrm{e}+01$ \\
\hline $\mathrm{OV}$ & 761.990 & $2 \mathrm{~s} 2 \mathrm{p}^{3} \mathrm{P}_{2}-2 \mathrm{p}^{2}{ }^{3} \mathrm{P}_{1}$ & & $1.165 \mathrm{e}+01$ & $1.446 \mathrm{e}+01$ & $9.213 e+01$ & $1.973 \mathrm{e}+02$ \\
\hline O VI & 1031.930 & $2 \mathrm{~s}^{2} \mathrm{~S}_{1 / 2}-2 \mathrm{p}^{2} \mathrm{P}_{3 / 2}$ & 5.47 & $1.117 \mathrm{e}+03$ & $1.080 \mathrm{e}+03$ & $2.917 \mathrm{e}+03$ & $1.097 \mathrm{e}+04$ \\
\hline O VI & 1037.640 & $2 \mathrm{~s}^{2} \mathrm{~S}_{1 / 2}-2 \mathrm{p}{ }^{2} \mathrm{P}_{1 / 2}$ & & $4.819 \mathrm{e}+02$ & $4.710 \mathrm{e}+02$ & $1.456 \mathrm{e}+03$ & $4.477 \mathrm{e}+03$ \\
\hline $\mathrm{Ne}$ VII & 895.170 & $2 \mathrm{~s}^{2}{ }^{1} \mathrm{~S}_{0}-2 \mathrm{~s} 2 \mathrm{p}{ }^{3} \mathrm{P}_{1}$ & 5.71 & $1.171 \mathrm{e}+01$ & $1.513 \mathrm{e}+01$ & $4.545 \mathrm{e}+01$ & $6.226 \mathrm{e}+01$ \\
\hline $\mathrm{Ne}$ VIII & 770.420 & $2 \mathrm{~s}^{2} \mathrm{~S}_{1 / 2}-2 \mathrm{p}^{2} \mathrm{P}_{3 / 2}$ & 5.80 & $1.202 \mathrm{e}+02$ & $1.398 \mathrm{e}+02$ & $3.742 \mathrm{e}+02$ & $2.751 \mathrm{e}+02$ \\
\hline $\mathrm{Ne}$ VIII & 780.300 & $2 \mathrm{~s}^{2} \mathrm{~S}_{1 / 2}-2 \mathrm{p}{ }^{2} \mathrm{P}_{1 / 2}$ & & $6.699 \mathrm{e}+01$ & $7.310 \mathrm{e}+01$ & $1.958 \mathrm{e}+02$ & $1.600 \mathrm{e}+02$ \\
\hline $\mathrm{Na} I X$ & 681.680 & $2{ }^{2} S_{1 / 2}-2 p^{2} P_{3 / 2}$ & 5.93 & $6.417 \mathrm{e}+00$ & $9.360 \mathrm{e}+00$ & $2.338 \mathrm{e}+01$ & $1.956 \mathrm{e}+01$ \\
\hline $\mathrm{Mg} \mathrm{V}$ & 1324.590 & $2 \mathrm{~s}^{2} 2 \mathrm{p}^{4}{ }^{3} \mathrm{P}_{1}-2 \mathrm{~s}^{2} 2 \mathrm{p}^{4}{ }^{1} \mathrm{~S}_{0}$ & 5.43 & $5.103 e+00$ & & & $2.056 \mathrm{e}+01$ \\
\hline Mg VI & 1191.680 & $2 s^{2} 2 p^{3}{ }^{4} S_{3 / 2}-2 s^{2} 2 p^{3}{ }^{2} P_{1 / 2}$ & 5.64 & $5.339 \mathrm{e}+00$ & & & $8.800 \mathrm{e}+00$ \\
\hline Mg VII & 868.130 & $2 \mathrm{~s}^{2} 2 \mathrm{p}^{2}{ }^{3} \mathrm{P}_{2}-2 \mathrm{~s} 2 \mathrm{p}^{3}{ }^{5} \mathrm{~S}_{2}$ & 5.80 & $6.372 \mathrm{e}+00$ & $7.056 \mathrm{e}+00$ & & $4.244 \mathrm{e}+01$ \\
\hline Mg VIII & 762.650 & $2 s^{2} 2 p^{2} P_{1 / 2}-2 s 2 p^{2}{ }^{4} P_{3 / 2}$ & 5.90 & $9.264 \mathrm{e}-01$ & $2.151 \mathrm{e}+00$ & $4.827 \mathrm{e}+00$ & $2.160 \mathrm{e}+00$ \\
\hline
\end{tabular}


H. Tian et al.: Solar transition region above sunspots, Online Material p 2

Table 5. continued.

\begin{tabular}{|c|c|c|c|c|c|c|c|}
\hline Ion & $\lambda_{\mathrm{obs}}$ & Transition & $\log \left(T_{f} / \mathrm{K}\right)$ & Umbra & Penumbra & Plage & Plume \\
\hline Mg VIII & 769.380 & $2 \mathrm{~s}^{2} 2 \mathrm{p}^{2} \mathrm{P}_{1 / 2}-2 \mathrm{~s} 2 \mathrm{p}^{2}{ }^{4} \mathrm{P}_{1 / 2}$ & & $1.888 \mathrm{e}+00$ & & & $3.403 e+00$ \\
\hline Mg VIII & 782.340 & $2 \mathrm{~s}^{2} 2 \mathrm{p}^{2} \mathrm{P}_{3 / 2}-2 \mathrm{~s} 2 \mathrm{p}^{2}{ }^{4} \mathrm{P}_{3 / 2}$ & & $5.775 e+00$ & $6.189 \mathrm{e}+00$ & $1.091 \mathrm{e}+01$ & $1.070 \mathrm{e}+01$ \\
\hline Mg VIII & 789.430 & $2 s^{2} 2 p^{2} P_{3 / 2}-2 s 2 p^{2}{ }^{4} P_{1 / 2}$ & & $2.818 \mathrm{e}+00$ & & & $4.980 \mathrm{e}+00$ \\
\hline $\mathrm{Mg}$ IX & 706.020 & $2 \mathrm{~s}^{2}{ }^{1} \mathrm{~S}_{0}-2 \mathrm{~s} 2 \mathrm{p}^{3} \mathrm{P}_{1}$ & 5.98 & $7.680 \mathrm{e}+00$ & $1.195 \mathrm{e}+01$ & $2.852 \mathrm{e}+01$ & $1.813 \mathrm{e}+01$ \\
\hline Mg IX & 749.540 & $2 \mathrm{~s} 2 \mathrm{p}{ }^{1} \mathrm{P}_{1}-2 \mathrm{p}^{2}{ }^{1} \mathrm{D}_{2}$ & & $1.534 \mathrm{e}+00$ & $2.893 e+00$ & $8.098 \mathrm{e}+00$ & $3.214 \mathrm{e}+00$ \\
\hline $\mathrm{Si}$ III & 1206.510 & $3^{\mathrm{s} 2{ }^{1}} \mathrm{~S}_{0}-3^{\mathrm{s} 3 \mathrm{p} 1} \mathrm{P}_{1}$ & 4.68 & $4.023 e+02$ & $2.882 \mathrm{e}+03$ & $2.488 \mathrm{e}+04$ & $1.853 e+03$ \\
\hline Si III & 1294.580 & $3^{s 3 p{ }^{3}} P_{1}-3 p^{2}{ }^{3} P_{2}$ & & $5.451 \mathrm{e}+00$ & $4.369 \mathrm{e}+01$ & $2.681 \mathrm{e}+02$ & $1.926 \mathrm{e}+01$ \\
\hline Si III & 1296.770 & $3^{s 3 p{ }^{3}} P_{0}-3 p^{2}{ }^{3} P_{1}$ & & $4.527 \mathrm{e}+00$ & $3.720 \mathrm{e}+01$ & $2.134 \mathrm{e}+02$ & $1.712 \mathrm{e}+01$ \\
\hline Si III & 1298.960 & $3^{s 3 p{ }^{3}} P_{2}-3 p^{2}{ }^{3} P_{2}$ & & $1.607 \mathrm{e}+01$ & $1.208 \mathrm{e}+02$ & $9.304 \mathrm{e}+02$ & $7.092 \mathrm{e}+01$ \\
\hline Si III & 1301.160 & $3^{\mathrm{s} 3 \mathrm{p}}{ }^{3} \mathrm{P}_{1}-3 \mathrm{p}^{2}{ }^{3} \mathrm{P}_{0}$ & & $3.316 \mathrm{e}+00$ & $3.012 \mathrm{e}+01$ & $1.898 \mathrm{e}+02$ & $1.488 \mathrm{e}+01$ \\
\hline Si IV & 815.050 & $3 p^{2} \mathrm{P}_{1 / 2}-4 s^{2} \mathrm{~S}_{1 / 2}$ & 4.86 & $1.086 \mathrm{e}+00$ & $4.641 \mathrm{e}+00$ & $1.105 \mathrm{e}+01$ & $3.598 \mathrm{e}+00$ \\
\hline Si IV & 818.150 & $3 \mathrm{p}^{2} \mathrm{P}_{3 / 2}-4 s^{2} \mathrm{~S}_{1 / 2}$ & & $2.399 \mathrm{e}+00$ & $1.048 \mathrm{e}+01$ & $2.952 \mathrm{e}+01$ & $9.645 e+00$ \\
\hline Si IV & 1128.350 & $3 p^{2} \mathrm{P}_{3 / 2}-3 \mathrm{~d}^{2} \mathrm{D}_{3 / 2,5 / 2}$ & & $1.399 \mathrm{e}+01$ & $5.594 \mathrm{e}+01$ & $1.893 e+02$ & $3.883 e+01$ \\
\hline Si IV & 1393.780 & $3^{s}{ }^{s} S_{1 / 2}-3 p^{2} P_{3 / 2}$ & & $4.358 \mathrm{e}+02$ & $3.648 \mathrm{e}+03$ & $1.580 \mathrm{e}+04$ & $1.468 \mathrm{e}+03$ \\
\hline Si IV & 1402.770 & $3^{s}{ }^{2} \mathrm{~S}_{1 / 2}-3 \mathrm{p}{ }^{2} \mathrm{P}_{1 / 2}$ & & $2.270 \mathrm{e}+02$ & $1.785 \mathrm{e}+03$ & $7.347 \mathrm{e}+03$ & $7.370 \mathrm{e}+02$ \\
\hline Si VII & 1049.250 & $2 \mathrm{~s}^{2} 2 \mathrm{p}^{4}{ }^{3} \mathrm{P}_{1}-2 \mathrm{~s}^{2} 2 \mathrm{p}^{4}{ }^{1} \mathrm{~S}_{0}$ & 5.76 & $2.474 \mathrm{e}+00$ & $2.128 \mathrm{e}+00$ & $3.247 \mathrm{e}+00$ & $1.895 \mathrm{e}+01$ \\
\hline Si IX & 676.490 & $2 \mathrm{~s}^{2} 2 \mathrm{p}^{2}{ }^{3} \mathrm{P}_{1}-2 \mathrm{~s} 2 \mathrm{p}^{3}{ }^{5} \mathrm{~S}_{2}$ & 6.06 & $6.827 \mathrm{e}-01$ & $1.047 \mathrm{e}+00$ & $3.022 \mathrm{e}+00$ & $1.750 \mathrm{e}+00$ \\
\hline S III & 1015.500 & $3^{\mathrm{s} 2} 3 \mathrm{p}^{2}{ }^{3} \mathrm{P}_{1}-3^{\mathrm{s} 3 \mathrm{p}^{3}} 3^{\mathrm{P0}, 1}$ & 4.66 & $6.887 \mathrm{e}-01$ & $2.669 \mathrm{e}+00$ & $8.193 e+00$ & $2.039 \mathrm{e}+00$ \\
\hline S III & 1015.770 & $3^{s 2} 3 p^{2}{ }^{3} P_{1}-3^{s 3 p 3} 3^{P 2}$ & & $5.016 \mathrm{e}-01$ & $2.445 \mathrm{e}+00$ & $6.699 e+00$ & $1.622 \mathrm{e}+00$ \\
\hline S III & 1021.080 & $3^{\mathrm{s} 2} 3 \mathrm{p}^{2}{ }^{3} \mathrm{P}_{2}-3^{\mathrm{s} 3 \mathrm{p} 3} 3^{\mathrm{P} 1}$ & & $4.251 \mathrm{e}-01$ & $2.649 \mathrm{e}+00$ & $6.981 \mathrm{e}+00$ & $1.149 \mathrm{e}+00$ \\
\hline S III & 1021.300 & $3^{\mathrm{s} 2} 3 \mathrm{p}^{2}{ }^{3} \mathrm{P}_{2}-3^{\mathrm{s} 3 \mathrm{p} 3} 3^{\mathrm{P} 2}$ & & $1.192 \mathrm{e}+00$ & $4.568 \mathrm{e}+00$ & $2.288 \mathrm{e}+01$ & $2.535 \mathrm{e}+00$ \\
\hline S III & 1200.990 & $3^{\mathrm{s} 2} 3 \mathrm{p}^{2}{ }^{3} \mathrm{P}_{2}-3^{\mathrm{s} 3 \mathrm{p} 3} 3^{\mathrm{D} 3}$ & & $5.086 e+00$ & $1.990 \mathrm{e}+01$ & $1.845 e+02$ & $1.516 \mathrm{e}+01$ \\
\hline S IV & 744.910 & $3^{\mathrm{s} 2} 3 \mathrm{p}^{2} \mathrm{P}_{1 / 2}-3^{\mathrm{s} 3 \mathrm{p} 2}{ }^{2} \mathrm{P}_{3 / 2}$ & 4.99 & $5.552 \mathrm{e}-01$ & $2.153 \mathrm{e}+00$ & $1.169 \mathrm{e}+01$ & $5.060 \mathrm{e}+00$ \\
\hline S IV & 748.400 & $3^{\mathrm{s} 2} 3 \mathrm{p}^{2} \mathrm{P}_{1 / 2}-3^{\mathrm{s} 3 \mathrm{p} 2}{ }^{2} \mathrm{P}_{1 / 2}$ & & $1.339 \mathrm{e}+00$ & $3.940 \mathrm{e}+00$ & $2.065 e+01$ & $1.176 \mathrm{e}+01$ \\
\hline S IV & 750.220 & $3^{\mathrm{s} 2} 3 \mathrm{p}^{2} \mathrm{P}_{3 / 2}-3^{\mathrm{s} 3 \mathrm{p} 2}{ }^{2} \mathrm{P}_{3 / 2}$ & & $2.766 \mathrm{e}+00$ & $8.431 \mathrm{e}+00$ & $5.318 \mathrm{e}+01$ & $2.622 \mathrm{e}+01$ \\
\hline S IV & 753.740 & $3^{s 2} 3 p^{2} P_{3 / 2}-3^{s 3 p 2}{ }^{2} P_{1 / 2}$ & & $8.233 \mathrm{e}-01$ & $2.544 \mathrm{e}+00$ & $1.245 \mathrm{e}+01$ & $6.416 \mathrm{e}+00$ \\
\hline S IV & 815.950 & $3^{\mathrm{s} 2} 3 \mathrm{p}^{2} \mathrm{P}_{3 / 2}-3^{\mathrm{s} 3 \mathrm{p} 2}{ }^{2} \mathrm{~S}_{1 / 2}$ & & $1.936 \mathrm{e}+00$ & $6.155 \mathrm{e}+00$ & $2.438 \mathrm{e}+01$ & $1.044 \mathrm{e}+01$ \\
\hline $\mathrm{S} \mathrm{V}$ & 786.470 & $3^{\mathrm{s} 2} \mathrm{~S}_{0}-3^{\mathrm{s} 3 \mathrm{p}{ }^{1}} \mathrm{P}_{1}$ & 5.19 & $2.781 \mathrm{e}+01$ & $4.254 \mathrm{e}+01$ & $2.257 \mathrm{e}+02$ & $5.116 \mathrm{e}+02$ \\
\hline S VI & 706.500 & $3 p^{2} P_{1 / 2}-3 d^{2} D_{3 / 2}$ & 5.29 & $1.020 \mathrm{e}+00$ & $1.667 \mathrm{e}+00$ & $2.746 \mathrm{e}+00$ & $5.460 \mathrm{e}+00$ \\
\hline Fe $\mathrm{X}$ & 1463.380 & $3^{s 2} 3 p^{4}\left({ }^{3} P\right) 3 d{ }^{4} F_{9 / 2}-3^{s 2} 3 p^{4}\left({ }^{1} D\right) 3 d^{2} F_{7 / 2}$ & 5.98 & $5.699 e+01$ & $2.279 \mathrm{e}+02$ & $4.276 \mathrm{e}+02$ & $1.147 \mathrm{e}+02$ \\
\hline Fe XII & 1241.950 & $3^{\mathrm{s} 2} 3 \mathrm{p}^{3}{ }^{4} \mathrm{~S}_{3 / 2}-3^{\mathrm{s} 2} 3 \mathrm{p}^{3}{ }^{2} \mathrm{P}_{3 / 2}$ & 6.13 & $3.325 \mathrm{e}+00$ & $2.596 \mathrm{e}+01$ & $9.015 e+01$ & $1.098 \mathrm{e}+01$ \\
\hline Fe XII & 1349.430 & $3^{\mathrm{s} 2} 3 \mathrm{p}^{3}{ }^{4} \mathrm{~S}_{3 / 2}-3^{\mathrm{s} 2} 3 \mathrm{p}^{3}{ }^{2} \mathrm{P}_{1 / 2}$ & & $1.605 \mathrm{e}+00$ & $1.551 \mathrm{e}+01$ & $5.158 \mathrm{e}+01$ & $8.580 \mathrm{e}+00$ \\
\hline
\end{tabular}


Table 6. List of "peculiar" lines identified in SUMER spectra of the sunspot. $A_{\mathrm{ki}}$ is the Einstein coefficient taken from NIST Atomic Spectral Database (Ralchenko et al. 2008) and Chianti Database (Dere et al. 1997; Landi et al. 2006). If available, unidentified lines are characterized by the temperature classification of Feldman et al. (1997) (a): $T_{\mathrm{e}}<3 \times 10^{5} \mathrm{~K}$, (b): $T_{\mathrm{e}} \approx 3 \times 10^{5} \mathrm{~K},(\mathrm{c}): T_{\mathrm{e}} \approx 4 \times 10^{5} \mathrm{~K},(\mathrm{~d}): 6 \times 10^{5} \mathrm{~K}<\mathrm{T}_{\mathrm{e}}<9 \times 10^{5} \mathrm{~K}$, (e): $T_{\mathrm{e}} \approx 1.4 \times 10^{6} \mathrm{~K}$, (f): $T_{\mathrm{e}} \approx 1.8 \times 10^{6} \mathrm{~K}$. The line intensities are given in the unit of $\mathrm{mW} \mathrm{sr}{ }^{-1} \mathrm{~m}^{-2}$. References in last column: (0) Curdt et al. (Curdt et al. (2001)), (1) Ekberg \& Feldman (Ekberg \& Feldman (2003)), (2) Sandlin et al. (Sandlin et al. (1986)) (3) New identification.

\begin{tabular}{|c|c|c|c|c|c|c|c|}
\hline$\lambda_{\text {obs }}(\AA)$ & Line & Intensity & & ansition & $A_{\mathrm{ki}}\left(\mathrm{s}^{-1}\right)$ & Also seen in & Reference \\
\hline 668.41 & (e) & 0.31800 & & & & streamer & 0 \\
\hline 669.01 & Al IX & 0.13913 & $2 s^{2} 2 p^{2} \mathrm{P}_{1 / 2}$ & $-2 \mathrm{~s} 2 \mathrm{p}^{2}{ }^{4} \mathrm{P}_{5 / 2}$ & $5.19 \mathrm{e}-02$ & streamer & 0 \\
\hline 670.02 & $\mathrm{Alx}$ & 0.23453 & $2 \mathrm{~s} 2 \mathrm{p}^{1} \mathrm{P}_{1}$ & $-2 \mathrm{p}^{2}{ }^{1} \mathrm{D}_{2}$ & $1.57 \mathrm{e}-03$ & streamer & 0 \\
\hline 672.55 & $?$ & 0.27117 & & & & & 0 \\
\hline 674.20 & $?$ & 0.34224 & & & & & 0 \\
\hline 679.78 & Mg VIII & 0.67506 & $2 \mathrm{~s} 2 \mathrm{p}^{2}{ }^{2} \mathrm{P}_{1 / 2}$ & $-2 p^{3}{ }^{2} D_{3 / 2}$ & $6.81 \mathrm{e}+08$ & weak in QS & 0 \\
\hline 680.39 & Al IX & 2.50337 & $2 s^{2} 2 p^{2} P_{1 / 2}$ & $-2 \mathrm{~s} 2 \mathrm{p}^{2}{ }^{4} \mathrm{P}_{3 / 2}$ & $3.84 \mathrm{e}+03$ & weak in QS & 0 \\
\hline 684.05 & $\mathrm{Na}$ VI & 0.37180 & $2 \mathrm{~s} 2 \mathrm{p}^{3}{ }^{3} \mathrm{D}_{3}$ & $-2 \mathrm{~s} 2 \mathrm{p}^{3}{ }^{1} \mathrm{P}_{1}$ & $7.28 \mathrm{e}-03$ & streamer & 3 \\
\hline 686.43 & Fe VIII & 2.02066 & $3 p^{5} 3 d^{2}{ }^{2} P_{3 / 2}$ & $-3 p^{6} 4 d^{2} D_{5 / 2}$ & $9.71 \mathrm{e}-01$ & & 1 \\
\hline 688.72 & Fe VIII & 0.17972 & $3 p^{5} 3 d^{2}{ }^{2} P_{3 / 2}$ & $-3 p^{6} 4 d^{2} D_{3 / 2}$ & $1.08 \mathrm{e}-01$ & & 1 \\
\hline 689.61 & Mg VIII & 0.81771 & $2 \mathrm{~s} 2 \mathrm{p}^{2}{ }^{2} \mathrm{P}_{3 / 2}$ & $-2 \mathrm{p}^{3}{ }^{2} \mathrm{D}_{5 / 2}$ & $7.51 \mathrm{e}+08$ & weak in QS & 0 \\
\hline 691.40 & CaIX & 5.52513 & $3^{\mathrm{s} 2}{ }^{1} \mathrm{~S}_{0}$ & $-3^{\mathrm{s} 3 \mathrm{p} 3} \mathrm{P}_{1}$ & $6.05 \mathrm{e}+05$ & corona & 0 \\
\hline 693.33 & $?$ & 0.37120 & & & & & 0 \\
\hline 694.70 & Si IX & 6.12387 & $2 \mathrm{~s}^{2} 2 \mathrm{p}^{2}{ }^{3} \mathrm{P}_{2}$ & $-2 \mathrm{~s} 2 \mathrm{p}^{3}{ }^{5} \mathrm{~S}_{2}$ & $1.38 \mathrm{e}+05$ & streamer & 0 \\
\hline 697.14 & Fe VIII & 4.56267 & $3 p^{6} 4 p^{2} P_{1 / 2}$ & $-3 p^{6} 4 d^{2} D_{3 / 2}$ & $2.00 \mathrm{e}+00$ & weak in QS & 1 \\
\hline 707.72 & (c) & 0.26568 & & & & & 0 \\
\hline 708.44 & (c) & 0.29286 & & & & & 0 \\
\hline 709.21 & Arv & 0.40630 & $3^{\mathrm{s} 2} 3 \mathrm{p}^{2}{ }^{3} \mathrm{P}_{1}$ & $-3^{\mathrm{s} 3 \mathrm{p} 3{ }^{3}} \mathrm{P}_{2}$ & & & 0 \\
\hline 712.89 & S VI & 4.34920 & $2 p^{6} 3 p^{2} P_{3 / 2}$ & $-2 p^{6} 3 d^{2} D_{5 / 2,3 / 2}$ & $8.10 \mathrm{e}+08$ & weak in QS & 0 \\
\hline 713.81 & Ar VIII & 4.92706 & $2 p^{6} 3^{s} S_{1 / 2}$ & $-2 p^{6} 3 p^{2} P_{1 / 2}$ & $2.40 \mathrm{e}+09$ & weak in QS & 0 \\
\hline 714.79 & $?$ & 0.22144 & & & & & 0 \\
\hline 715.65 & Arv & 0.81987 & $3^{\mathrm{s} 2} 3 \mathrm{p}^{2}{ }^{3} \mathrm{P}_{2}$ & $-3^{\mathrm{s} 3 \mathrm{p} 3{ }^{3}} \mathrm{P}_{1}$ & & weak in QS & 0 \\
\hline 717.01 & $?$ & 0.53082 & & & & corona & 0 \\
\hline 717.69 & Fe VIII ? & 0.31754 & $3 p^{6} 4 p^{2} P_{3 / 2}$ & $-3 p^{6} 4 d^{2} D_{5 / 2}$ & & & 0 \\
\hline 719.35 & S IX & 0.24769 & $2 \mathrm{p}^{3} 3 \mathrm{p}\left({ }^{2} \mathrm{D}\right)^{3} \mathrm{~F}_{4}$ & ${ }_{4}-2 p^{3} 3 d\left({ }^{3} D\right)^{3} G_{5}$ & $2.36 \mathrm{e}+09$ & corona & 0 \\
\hline 721.23 & Fe VIII & 8.42192 & $3 p^{6} 4 p^{2} \mathrm{P}_{1 / 2}$ & $-3 p^{6} 4 d^{2} D_{3 / 2}$ & $2.80 \mathrm{e}+00$ & weak in QS & 1 \\
\hline 723.75 & Fe VIII & 0.65898 & $3 p^{6} 4 p^{2} P_{3 / 2}$ & $-3 p^{6} 4 d^{2} D_{3 / 2}$ & $3.10 \mathrm{e}-01$ & & 1 \\
\hline 725.11 & Ar V & 0.64130 & $3^{s 2} 3 p^{2}{ }^{1} D_{2}$ & $-3^{\mathrm{s} 3 \mathrm{p} 3{ }^{1}} \mathrm{D}_{2}$ & & weak in QS & 0 \\
\hline 728.11 & Fe VII & 0.41838 & $3 d 4 p{ }^{3} D_{2}$ & $-3 d 4 d{ }^{3} F_{3}$ & & & 1 \\
\hline 735.42 & Fe VII & 0.39913 & $3 \mathrm{~d} 4 \mathrm{p}{ }^{3} \mathrm{P}_{1}$ & $-3 d 4 d{ }^{3} P_{2}$ & & & 1 \\
\hline 736.71 & $?$ & 0.78293 & & & & & 0 \\
\hline 738.87 & Fe VII & 0.80524 & $3 \mathrm{~d} 4 \mathrm{p}{ }^{3} \mathrm{P}_{2}$ & $-3 d 4 d{ }^{3} P_{2}$ & & & 1 \\
\hline 740.03 & Fe VII & & $3 \mathrm{~d} 4 \mathrm{p}{ }^{1} \mathrm{~F}_{3}$ & $-3 \mathrm{~d} 4 \mathrm{~d}{ }^{1} \mathrm{G}_{4}$ & & & 1 \\
\hline 740.11 & Ar VIII & & $2 p^{6} 4 d^{2} D_{5 / 2}$ & $-2 \mathrm{p}^{6} 5 p^{2} \mathrm{P}_{3 / 2}$ & & & 0 \\
\hline 740.79 & Fe VII & 0.84513 & $3 d 4 p{ }^{3} D_{3}$ & $-3 \mathrm{~d} 4 \mathrm{~d}^{3} \mathrm{~F}_{4}$ & & & 1 \\
\hline 741.14 & Fe VII & 0.66444 & $3 \mathrm{~d} 4 \mathrm{p}{ }^{3} \mathrm{P}_{1}$ & $-3 d 4 d{ }^{3} P_{0}$ & & & 1 \\
\hline 745.38 & Fe VII & 0.68352 & $3 d 4 p^{3} F_{2}$ & $-3 d 4 d{ }^{3} F_{2}$ & & & 1 \\
\hline 747.37 & $?$ & 0.55478 & & & & & 0 \\
\hline 749.01 & Fe VII & 1.15036 & $3 d 4 p{ }^{3} F_{3}$ & $-3 d 4 d{ }^{3} F_{3}$ & & & 1 \\
\hline 750.58 & (a) & 1.38937 & & & & streamer & 0 \\
\hline 751.46 & (a) & 0.53180 & & & & streamer & 0 \\
\hline 752.51 & Fe VIII & 0.65775 & $3 p^{5} 3 d^{2}{ }^{2} P_{1 / 2}$ & $-3 p^{6} 4 d^{2} D_{3 / 2}$ & & & 1 \\
\hline 753.22 & Mg VIII & 1.08143 & $2 \mathrm{~s}^{2} 2 \mathrm{p}^{2} \mathrm{P}_{1 / 2}$ & $-2 \mathrm{~s} 2 \mathrm{p}^{2}{ }^{4} \mathrm{D}_{5 / 2}$ & $3.46 \mathrm{e}-02$ & corona & 3 \\
\hline 754.93 & Ar VI & 4.15884 & $3^{s 2} 3 p^{2} \mathrm{P}_{1 / 2}$ & $-3^{\mathrm{s} 3 \mathrm{p}^{2}}{ }^{2} \mathrm{D}_{3 / 2}$ & $2.80 \mathrm{e}+09$ & & 0 \\
\hline 756.71 & Al VIII & 0.87528 & $2 \mathrm{~s}^{2} 2 \mathrm{p}^{2}{ }^{3} \mathrm{P}_{1}$ & $-2 \mathrm{~s} 2 \mathrm{p}^{3}{ }^{5} \mathrm{~S}_{2}$ & $3.00 \mathrm{e}+04$ & & 0 \\
\hline 757.15 & Fe VII & 2.65446 & $3 d 4 p^{3} F_{4}$ & $-3 d 4 d^{3} F_{4}$ & & & 1 \\
\hline 762.65 & Mg VIII & 1.77679 & $2 s^{2} 2 p^{2} P_{1 / 2}$ & $-2 \mathrm{~s} 2 \mathrm{p}^{2}{ }^{4} \mathrm{P}_{3 / 2}$ & $1.71 \mathrm{e}+03$ & corona & 0 \\
\hline 767.07 & ArVI & 9.64480 & $3^{\mathrm{s} 2} 3 \mathrm{p}^{2} \mathrm{P}_{3 / 2}$ & $-3^{\mathrm{s} 3 \mathrm{p}^{2} 2} \mathrm{D}_{5 / 2}$ & $3.30 \mathrm{e}+09$ & weak in QS & 0 \\
\hline 769.38 & Mg VIII & 6.67460 & $2 s^{2} 2 p^{2} P_{1 / 2}$ & $-2 \mathrm{~s} 2 \mathrm{p}^{2}{ }^{4} \mathrm{P}_{1 / 2}$ & $7.29 \mathrm{e}+04$ & corona & 0 \\
\hline 772.26 & Mg VIII & 43.7776 & $2 s^{2} 2 p^{2} P_{3 / 2}$ & $-2 \mathrm{~s} 2 \mathrm{p}^{2}{ }^{4} \mathrm{P}_{5 / 2}$ & $5.67 \mathrm{e}+04$ & corona & 0 \\
\hline 776.62 & (b) & 3.69754 & & & & streamer & 0 \\
\hline 782.34 & Mg VIII & 7.38377 & $2 s^{2} 2 p{ }^{2} P_{3 / 2}$ & $-2 \mathrm{~s} 2 \mathrm{p}^{2}{ }^{4} \mathrm{P}_{3 / 2}$ & $1.61 \mathrm{e}+04$ & corona & 0 \\
\hline 784.52 & Fe VII & 2.02086 & $3 d 4 p^{3} F_{1}$ & $-3 d 4 d^{3} S_{1}$ & & & 1 \\
\hline 789.43 & Mg VIII & 5.96976 & $2 s^{2} 2 p^{2} P_{3 / 2}$ & $-2 \mathrm{~s} 2 \mathrm{p}^{2}{ }^{4} \mathrm{P}_{1 / 2}$ & $5.96 \mathrm{e}+04$ & corona & 0 \\
\hline 789.78 & Na VIII & 4.45572 & $2 \mathrm{~s}^{2}{ }^{1} \mathrm{~S}_{0}$ & $-2 \mathrm{~s} 2 \mathrm{p}^{3} \mathrm{P}_{1}$ & $3.63 \mathrm{e}+04$ & & 0 \\
\hline 792.77 & Fe VII & 3.80994 & $3 d 4 p{ }^{3} F_{2}$ & $-3 d 4 d^{3} G_{3}$ & & & 1 \\
\hline 793.75 & $?$ & 1.54882 & & & & & 3 \\
\hline 794.25 & $?$ & 1.28655 & & & & & 3 \\
\hline 795.23 & (a) & 1.54914 & & & & streamer & 0 \\
\hline 799.11 & Fe VII & 1.73620 & $3 d 4 p^{3} D_{3}$ & $-3 d 4 d{ }^{3} D_{3}$ & & & 1 \\
\hline
\end{tabular}


Table 6. continued.

\begin{tabular}{|c|c|c|c|c|c|c|}
\hline$\lambda_{\mathrm{obs}}(\AA)$ & Line & Intensity & Transition & $A_{\mathrm{ki}}\left(\mathrm{s}^{-1}\right)$ & Also seen in & Reference \\
\hline 803.46 & (e) & 3.26169 & & & streamer & 0 \\
\hline 804.16 & Fe VII & 8.47300 & $-3 \mathrm{~d} 4 \mathrm{~d}^{3} \mathrm{G}_{5}$ & & & 1 \\
\hline 811.55 & Mn VII & 1.67024 & $3 p^{6} 4 p^{2} P_{3 / 2}-3 p^{6} 4 d^{2} D_{5 / 2}$ & & & 3 \\
\hline 813.01 & $\mathrm{Cl}$ VII & 2.43765 & $2 p^{6} 3^{s} S_{1 / 2}-2 p^{6} 3 p^{2} P_{1 / 2}$ & $2.11 \mathrm{e}+09$ & & 0 \\
\hline 821.23 & Ca IX & 3.87217 & $3^{33 p^{1} \mathrm{P}_{1}} \quad-3 \mathrm{p}^{2}{ }^{1} \mathrm{D}_{2}$ & $6.79 \mathrm{e}+08$ & & 0 \\
\hline 827.06 & ArV & 2.10911 & $-3^{\mathrm{s} 3 \mathrm{p} 3{ }^{3}} \mathrm{D}_{2}$ & $2.20 \mathrm{e}+09$ & & 0 \\
\hline 849.29 & S V & 4.01087 & $-3 p^{2}{ }^{3} P_{2}$ & $1.07 \mathrm{e}+09$ & & 0 \\
\hline 852.17 & S v & 5.69634 & $3^{\mathrm{s} 3 \mathrm{p}} \mathrm{P}_{0} \quad-3 \mathrm{p}^{2}{ }^{3} \mathrm{P}_{1}$ & $1.50 \mathrm{e}+09$ & & 0 \\
\hline 854.71 & Mg VII & & $2 s^{2} 2 p^{2}{ }^{3} P_{1}-2 s 2 p^{3}{ }^{5} S_{2}$ & $1.14 \mathrm{e}+04$ & & 0 \\
\hline 854.80 & S V & & $3^{33 p^{3} P_{2}} \quad-3 p^{2}{ }^{3} P_{2}$ & $3.13 \mathrm{e}+09$ & & 0 \\
\hline$\overline{8} 57.82$ & S V & 2.94460 & $-3 \mathrm{p}^{2}{ }^{3} \mathrm{P}_{0}$ & $4.14 \mathrm{e}+09$ & & 0 \\
\hline 860.48 & S V & 6.74638 & $-3 p^{2}{ }^{3} P_{1}$ & $1.71 \mathrm{e}+09$ & & 0 \\
\hline 868.13 & Mg VII & 44.0762 & $2 \mathrm{~s}^{2} 2 \mathrm{p}^{2}{ }^{3} \mathrm{P}_{2}-2 \mathrm{~s} 2 \mathrm{p}^{3}{ }^{5} \mathrm{~S}_{2}$ & $2.90 \mathrm{e}-02$ & & 0 \\
\hline 872.12 & Na VII & 9.19926 & $2 s^{2} 2 p^{2} P_{3 / 2}-2 s 2 p^{2}{ }^{4} P_{5 / 2}$ & $2.84 \mathrm{e}+04$ & & 0 \\
\hline 873.78 & Siv? & 6.17296 & $2 p^{5} 3^{s}{ }^{1} P_{1} \quad-2 p^{5} 3 p{ }^{1} S_{0}$ & $5.06 e+09$ & streamer & 3 \\
\hline 877.92 & Ar VII ? & 7.16439 & $-3^{33 p{ }^{3}} P_{1}$ & & & 0 \\
\hline 880.33 & Na VII & 3.85727 & $2 \mathrm{~s}^{2} 2 \mathrm{p}^{2} \mathrm{P}_{3 / 2}-2 \mathrm{~s} 2 \mathrm{p}^{2}{ }^{4} \mathrm{P}_{3 / 2}$ & $7.77 \mathrm{e}+03$ & & 0 \\
\hline 885.33 & (b) & 5.53839 & & & streamer & 0 \\
\hline 895.17 & Ne VII & 80.3477 & $-2 \mathrm{~s} 2 \mathrm{p}{ }^{3} \mathrm{P}_{1}$ & $1.32 \mathrm{e}+04$ & weak in QS & 0 \\
\hline 905.02 & Si VII & 8.35475 & $2 p^{3} 3 p^{5} P_{2}-2 p^{3} 3 d^{5} D_{2,3}$ & $1.22 \mathrm{e}+09$ & & 0 \\
\hline 944.34 & Si VIII & 33.6785 & $2 s^{2} 2 p^{3}{ }^{4} S_{3 / 2}-2 s^{2} 2 p^{3}{ }^{2} P_{3 / 2}$ & $7.23 \mathrm{e}+01$ & weak in QS & 0 \\
\hline 957.11 & O vi? & 1.59753 & & & streamer & 0 \\
\hline 965.28 & (d) & 1.18173 & & & streamer & 0 \\
\hline 966.68 & $?$ & 0.92318 & & & & 0 \\
\hline 975.85 & (d) & 0.96417 & & & streamer & 0 \\
\hline 997.21 & $\mathrm{Ne}$ VI & 11.5087 & $2 \mathrm{~s}^{2} 2 \mathrm{p}^{2} \mathrm{P}_{1 / 2}-2 \mathrm{~s} 2 \mathrm{p}^{2}{ }^{4} \mathrm{P}_{1 / 2}$ & $1.18 \mathrm{e}+04$ & & 0 \\
\hline 998.05 & $?$ & 2.18132 & & & & 0 \\
\hline 999.25 & $\mathrm{Ne} \mathrm{VI}$ & 62.3252 & $2 \mathrm{~s}^{2} 2 \mathrm{p}^{2} \mathrm{P}_{3 / 2}-2 \mathrm{~s} 2 \mathrm{p}^{2}{ }^{4} \mathrm{P}_{5 / 2}$ & $9.08 \mathrm{e}+03$ & weak in QS & 0 \\
\hline 1005.79 & $\mathrm{Ne} \mathrm{VI}$ & 32.5323 & $2 s^{2} 2 p^{2} P_{3 / 2}-2 s 2 p^{2}{ }^{4} P_{3 / 2}$ & $2.92 \mathrm{e}+03$ & & 0 \\
\hline 1010.25 & $\mathrm{Ne} \mathrm{VI}$ & 12.4947 & $2 \mathrm{~s}^{2} 2 \mathrm{p}^{2} \mathrm{P}_{3 / 2}-2 \mathrm{~s} 2 \mathrm{p}^{2}{ }^{4} \mathrm{P}_{1 / 2}$ & $1.06 \mathrm{e}+04$ & & 0 \\
\hline 1011.61 & $?$ & 1.31622 & & & & 0 \\
\hline 1012.65 & $?$ & 1.96463 & & & & 0 \\
\hline 1013.88 & $?$ & 1.26811 & & & & 0 \\
\hline 1028.95 & (e) & 3.54614 & & & streamer & 0 \\
\hline 1049.25 & Si VII & 12.4464 & $2 s^{2} 2 p^{4}{ }^{3} P_{1}-2 s^{2} 2 p^{4}{ }^{1} S_{0}$ & $1.39 \mathrm{e}+02$ & & 0 \\
\hline 1051.60 & $\mathrm{O} \mathrm{III} / 2$ & 33.8099 & $2 \mathrm{~s}^{2} 2 \mathrm{p}^{2}{ }^{1} \mathrm{D}_{2}-2 \mathrm{~s} 2 \mathrm{p}^{3}{ }^{1} \mathrm{P}_{1}$ & $9.60 \mathrm{e}+09$ & & 0 \\
\hline 1053.87 & Al VII & 4.47774 & $2 s^{2} 2 p^{3}{ }^{4} S_{3 / 2}-2 s^{2} 2 p^{3}{ }^{2} P_{3 / 2}$ & $3.13 \mathrm{e}+01$ & & 0 \\
\hline 1056.81 & Al VII & 2.29385 & $2 \mathrm{~s}^{2} 2 \mathrm{p}^{3}{ }^{4} \mathrm{~S}_{3 / 2}-2 \mathrm{~s}^{2} 2 \mathrm{p}^{3}{ }^{2} \mathrm{P}_{1 / 2}$ & $1.28 \mathrm{e}+01$ & weak in QS & 0 \\
\hline 1057.81 & Al VIII & 1.73163 & $2 \mathrm{~s}^{2} 2 \mathrm{p}^{2}{ }^{3} \mathrm{P}_{1}-2 \mathrm{~s}^{2} 2 \mathrm{p}^{2}{ }^{1} \mathrm{~S}_{0}$ & $9.05 \mathrm{e}+01$ & & 0 \\
\hline 1067.83 & O IV & 3.86110 & $2 \mathrm{~s}^{2} 3 \mathrm{~d}^{2} \mathrm{D}_{5 / 2}-2 \mathrm{~s}^{2} 4 f^{2} \mathrm{~F}_{7 / 2}$ & $3.33 \mathrm{e}+09$ & & 0 \\
\hline 1087.86 & $\mathrm{Ne} \mathrm{IV} / 2$ & & $2 s^{2} 2 p^{3}{ }^{3} S_{3 / 2}-2 s 2 p^{4}{ }^{4} P_{5 / 2}$ & $2.50 \mathrm{e}+09$ & & 3 \\
\hline 1087.86 & Fe VII & & $3 \mathrm{~d} 4 s^{3} \mathrm{D}_{2}-3 \mathrm{~d} 4 \mathrm{p}^{3} \mathrm{P}_{1}$ & & & 1 \\
\hline 1095.37 & Fe VII & 3.09757 & $-3 d 4 p{ }^{3} P_{2}$ & & & 1 \\
\hline 1098.92 & $?$ & 2.72720 & & & & 0 \\
\hline 1106.70 & $\mathrm{O} \mathrm{IV} / 2$ & 169.437 & $2 \mathrm{~s}^{2} 2 \mathrm{p}^{2} \mathrm{P}_{1 / 2}-2 \mathrm{~s} 2 \mathrm{p}^{2}{ }^{2} \mathrm{P}_{3 / 2}$ & $1.22 \mathrm{e}+09$ & weak in QS & 0 \\
\hline 1108.16 & $\mathrm{O} \mathrm{IV} / 2$ & 195.290 & $2 s^{2} 2 p^{2} P_{1 / 2}-2 s 2 p^{2}{ }^{2} P_{1 / 2}$ & $4.86 \mathrm{e}+09$ & weak in QS & 0 \\
\hline 1109.12 & $\mathrm{O} \mathrm{IV} / 2$ & 685.028 & $2 s^{2} 2 p^{2} P_{3 / 2}-2 s 2 p^{2}{ }^{2} P_{3 / 2}$ & $6.06 e+09$ & weak in QS & 0 \\
\hline 1110.52 & $\mathrm{O} \mathrm{IV} / 2$ & 132.044 & $2 \mathrm{~s}^{2} 2 \mathrm{p}^{2} \mathrm{P}_{3 / 2}-2 \mathrm{~s} 2 \mathrm{p}^{2}{ }^{2} \mathrm{P}_{1 / 2}$ & $2.41 \mathrm{e}+09$ & weak in QS & 0 \\
\hline 1115.52 & $\mathrm{CaX} / 2$ & 49.6308 & $2 p^{6} 3^{5} S_{1 / 2}-2 p^{6} 3 p^{2} P_{3 / 2}$ & $3.50 \mathrm{e}+09$ & & 0 \\
\hline 1119.10 & $\mathrm{H}_{2}$ & 113.679 & $1-3 Q 3 \quad(C-X)$ & & & 0 \\
\hline 1125.66 & $\mathrm{Ne} \mathrm{VI} / 2$ & 343.100 & $2 s^{2} 2 p^{2} P_{3 / 2}-2 s 2 p^{2}{ }^{2} D_{5 / 2,3 / 2}$ & $1.17 \mathrm{e}+09$ & weak in QS & 0 \\
\hline 1128.35 & Si IV & 36.3423 & $2 p^{6} 3 p^{2} P_{3 / 2}-2 p^{6} 3 d^{2} D_{5 / 2,3 / 2}$ & $2.53 \mathrm{e}+09$ & weak in QS & 0 \\
\hline 1136.56 & $\mathrm{Ne} V$ & 19.1372 & $2 \mathrm{~s}^{2} 2 \mathrm{p}^{2}{ }^{3} \mathrm{P}_{1}-2 \mathrm{~s} 2 \mathrm{p}^{3}{ }^{5} \mathrm{~S}_{2}$ & $2.23 e+03$ & weak in QS & 0 \\
\hline 1136.82 & $\mathrm{Ne} / / 2$ & 47.5348 & $2 s^{2} 2 p^{2}{ }^{3} P_{0}-2 s 2 p^{3}{ }^{3} D_{1}$ & $7.11 \mathrm{e}+08$ & & 0 \\
\hline 1141.45 & Fe VII & 5.09396 & $3 \mathrm{~d} 4 s^{3} \mathrm{D}_{3} \quad-3 \mathrm{~d} 4 \mathrm{p}^{3} \mathrm{~F}_{4}$ & & & 1 \\
\hline 1144.28 & $\mathrm{Ne} / 2$ & 15.2854 & $2 s^{2} 2 p^{2}{ }^{3} P_{2}-2 s 2 p^{3}{ }^{3} D_{1}$ & $2.88 \mathrm{e}+07$ & & 0 \\
\hline 1144.74 & $\mathrm{Ne}$ V/2 & 101.738 & $2 s^{2} 2 p^{2}{ }^{3} P_{2}-2 s 2 p^{3}{ }^{3} D_{3}$ & $2.76 \mathrm{e}+08$ & & 0 \\
\hline 1145.66 & $\mathrm{NeV}$ & 33.2632 & $2 s^{2} 2 p^{2}{ }^{3} P_{2}-2 s 2 p^{3}{ }^{5} s_{2}$ & $6.38 \mathrm{e}+03$ & weak in QS & 0 \\
\hline 1148.06 & $\mathrm{CaX} / 2$ & 23.1761 & $2 p^{6} 3^{s} S_{1 / 2}-2 p^{6} 3 p^{2} P_{1 / 2}$ & $3.20 \mathrm{e}+09$ & & 0 \\
\hline 1149.59 & (d) & 1.34829 & & & & 3 \\
\hline 1155.01 & Fe VII & 0.52653 & $3 \mathrm{~d} 4 s^{3} \mathrm{D}_{2} \quad-3 \mathrm{~d} 4 \mathrm{p}^{3} \mathrm{~F}_{3}$ & & & 1 \\
\hline 1163.85 & $\mathrm{H}_{2}$ & 180.646 & $1-4 Q 3 \quad(C-X)$ & & & 0 \\
\hline 1165.74 & $\mathrm{Ca} \mathrm{VIII/2}$ & 41.3746 & $3^{\mathrm{s} 2} 3 \mathrm{p}^{2} \mathrm{P}_{1 / 2}-3^{\mathrm{s} 3 \mathrm{p}^{2}}{ }^{2} \mathrm{D}_{3 / 2}$ & $4.10 \mathrm{e}+09$ & & 0 \\
\hline 1166.22 & Fe VII & 2.91614 & $3 \mathrm{~d} 4 s^{3} \mathrm{D}_{1} \quad-3 \mathrm{~d} 4 \mathrm{p}^{3} \mathrm{~F}_{2}$ & & & 1 \\
\hline 1170.18 & $?$ & 10.1514 & & & & 0 \\
\hline
\end{tabular}


H. Tian et al.: Solar transition region above sunspots, Online Material p 5

Table 6. continued.

\begin{tabular}{|c|c|c|c|c|c|c|c|}
\hline$\lambda_{\mathrm{obs}}(\AA)$ & Line & Intensity & & Transition & $A_{\mathrm{ki}}\left(\mathrm{s}^{-1}\right)$ & Also seen in & Reference \\
\hline 1171.60 & Ar VII/2 & 84.1832 & $3^{\mathrm{s} 2}{ }^{1} \mathrm{~S}_{0}$ & $-3^{\mathrm{s} 3 \mathrm{p} 1} \mathrm{P}_{1}$ & $7.83 e+09$ & & 0 \\
\hline 1189.84 & Mg VII & 27.2145 & $2 \mathrm{~s}^{2} 2 \mathrm{p}^{2}{ }^{3} \mathrm{P}_{1}$ & $-2 \mathrm{~s}^{2} 2 \mathrm{p}^{2}{ }^{1} \mathrm{~S}_{0}$ & $3.62 \mathrm{e}+01$ & & 0 \\
\hline 1190.12 & Mg VI & 123.208 & $2 s^{2} 2 p^{3}{ }^{4} S_{3 / 2}$ & $-2 \mathrm{~s}^{2} 2 \mathrm{p}^{3}{ }^{2} \mathrm{P}_{3 / 2}$ & $1.21 \mathrm{e}+01$ & & 0 \\
\hline 1191.68 & Mg VI & 30.3776 & $2 s^{2} 2 p^{3}{ }^{4} S_{3 / 2}$ & $-2 \mathrm{~s}^{2} 2 \mathrm{p}^{3}{ }^{2} \mathrm{P}_{1 / 2}$ & $4.91 \mathrm{e}+00$ & & 0 \\
\hline 1199.21 & S V & 91.5734 & $3^{\mathrm{s} 2}{ }^{1} \mathrm{~S}_{0}$ & $-3^{\mathrm{s} 3 \mathrm{p}^{3}} \mathrm{P}_{1}$ & $1.23 \mathrm{e}+05$ & weak in QS & 0 \\
\hline 1209.04 & $\mathrm{H}_{2}$ & 121.340 & $1-5 Q 3 \quad(C-X)$ & & & & 0 \\
\hline 1218.34 & $\mathrm{OV}$ & 2085.46 & $2 \mathrm{~s}^{2}{ }^{1} \mathrm{~S}_{0}$ & $-2 \mathrm{~s} 2 \mathrm{p}^{3} \mathrm{P}_{1}$ & $2.34 \mathrm{e}+03$ & & 0 \\
\hline 1251.48 & Siv & 7.48967 & $2 s^{2} 2 p^{5} 3 p^{3} P_{2}$ & $-2 s^{2} 2 p^{5} 3 p^{3} D_{3}$ & $9.59 \mathrm{e}+08$ & & 3 \\
\hline 1251.76 & Si V & 4.77757 & $2 s^{2} 2 p^{5} 3 p^{1} P_{1}$ & $-2 s^{2} 2 p^{5} 3 p^{3} P_{1}$ & $3.96 \mathrm{e}+08$ & & 3 \\
\hline 1254.11 & $\mathrm{H}_{2}$ & 17.6018 & $1-6 Q 3 \quad(C-X)$ & & & & 0 \\
\hline 1257.24 & $\mathrm{H}_{2}$ & 2.94243 & $1-3 R 3 \quad(X-B)$ & & & & 2 \\
\hline 1267.76 & $\mathrm{Ca} v \mathrm{vi} / 2$ & 14.2663 & $3^{s 2} 3 p^{3}{ }^{4} S_{3 / 2}$ & $-3^{\mathrm{s} 3 \mathrm{p} 4}{ }^{4} \mathrm{P}_{3 / 2}$ & $5.00 \mathrm{e}+09$ & & 0 \\
\hline 1283.92 & $\mathrm{Ca} \mathrm{vI} / 2$ & 29.4227 & $3^{s 2} 3 p^{3}{ }^{4} S_{3 / 2}$ & $-3^{\mathrm{s} 3 \mathrm{p} 4{ }^{4}} \mathrm{P}_{5 / 2}$ & $4.70 \mathrm{e}+09$ & & 0 \\
\hline 1285.47 & $\mathrm{Siv}$ & 2.21361 & $2 s^{2} 2 p^{5} 3^{s}{ }^{3} P_{0}$ & $-2 s^{2} 2 p^{5} 3 p^{3} D_{1}$ & $2.67 \mathrm{e}+08$ & & 3 \\
\hline 1285.68 & $\mathrm{H}_{2}$ & 1.85936 & $1-3 P 7 \quad(X-B)$ & & & & 0 \\
\hline 1286.20 & $\mathrm{Cav} / 2$ & 4.63060 & $3^{\mathrm{s} 2} 3 \mathrm{p}^{4} \mathrm{P}_{1}$ & $-3^{\mathrm{s} 3 \mathrm{p} 5{ }^{3}} \mathrm{P}_{0}$ & $9.10 \mathrm{e}+08$ & weak in QS & 0 \\
\hline 1286.43 & $\mathrm{H}_{2}$ & 1.19117 & $3-4$ P5 $\quad(X-B)$ & & & & 0 \\
\hline 1293.14 & $\mathrm{Cav} / 2$ & 4.54793 & $3^{\mathrm{s} 2} 3 \mathrm{p}^{4}{ }^{3} \mathrm{P}_{2}$ & $-3^{\mathrm{s} 3 \mathrm{p} 5{ }^{3}} \mathrm{P}_{2}$ & $6.90 \mathrm{e}+08$ & & 0 \\
\hline 1293.88 & $\mathrm{Mg} \mathrm{V}$ & 0.69026 & $2 s^{2} 2 p^{4}{ }^{3} \mathrm{P}_{2}$ & $-2 \mathrm{~s}^{2} 2 \mathrm{p}^{4}{ }^{1} \mathrm{~S}_{0}$ & & & 3 \\
\hline 1314.67 & $\mathrm{~S} \mathrm{IV} / 2$ & 20.7701 & $3^{\mathrm{s} 2} 3 \mathrm{p}^{2} \mathrm{P}_{1 / 2}$ & $-3^{\mathrm{s} 2} 3 \mathrm{~d}^{2} \mathrm{D}_{3 / 2}$ & $8.69 e+09$ & weak in QS & 0 \\
\hline 1317.84 & $?$ & 1.43195 & & & & & 0 \\
\hline 1319.76 & $\mathrm{~S} v / 2$ & 9.49761 & $3^{\mathrm{s} 3 \mathrm{p}{ }^{3} \mathrm{P}_{1}}$ & $-3^{\mathrm{s} 3 \mathrm{~d} 3} \mathrm{D}_{2,1}$ & $4.87 e+09$ & weak in QS & 0 \\
\hline 1322.84 & $\mathrm{~S}$ IV/2 & 34.1715 & $3^{\mathrm{s} 2} 3 \mathrm{p}^{2} \mathrm{P}_{3 / 2}$ & $-3^{\mathrm{s} 2} 3 \mathrm{~d}^{2} \mathrm{D}_{5 / 2,3 / 2}$ & $1.03 \mathrm{e}+10$ & weak in QS & 0 \\
\hline 1324.59 & $\mathrm{Mg} \mathrm{V}$ & 30.8036 & $2 s^{2} 2 p^{4}{ }^{3} P_{1}$ & $-2 \mathrm{~s}^{2} 2 \mathrm{p}^{4}{ }^{1} \mathrm{~S}_{0}$ & $2.15 \mathrm{e}+01$ & & 0 \\
\hline 1326.36 & $\mathrm{~S} \mathrm{~V} / 2$ & 16.7918 & $3^{\mathrm{s} 3 \mathrm{p}{ }^{3}} \mathrm{P}_{2}$ & $-3^{\mathrm{s} 3 \mathrm{~d}}{ }^{3} D_{3,2}$ & $6.39 \mathrm{e}+09$ & & 0 \\
\hline 1338.57 & $\mathrm{H}_{2}$ & 3.96306 & $0-4 P 2 \quad(X-B)$ & & & & 0 \\
\hline 1339.42 & $\mathrm{Al} \mathrm{v} / 2$ & 3.40516 & $2 s^{2} 2 p^{4}\left({ }^{3} P\right) 3 d^{4} F_{7 /}$ & $7 / 2-2 \mathrm{~s}^{2} 2 \mathrm{p}^{4}\left({ }^{3} \mathrm{P}_{2}\right) 4 f^{2}[4]_{7 / 2}$ & & & 3 \\
\hline 1342.88 & $\mathrm{Cl} \mathrm{VI} / 2$ & 6.51297 & $2 \mathrm{p}^{6} 3^{\mathrm{s} 2}{ }^{1} \mathrm{~S}_{0}$ & $-3{ }^{\mathrm{s} 3 \mathrm{p}{ }^{1}} \mathrm{P}_{1}$ & $6.32 \mathrm{e}+09$ & & 3 \\
\hline 1343.64 & $?$ & 4.24962 & & & & & 0 \\
\hline 1347.06 & $?$ & 4.15148 & & & & & 0 \\
\hline 1348.79 & $?$ & 2.67438 & & & & & 0 \\
\hline 1349.43 & Fe XII & 10.4184 & $3^{\mathrm{s} 2} 3 p^{3}{ }^{4} S_{3 / 2}$ & $-3^{\mathrm{s} 2} 3 \mathrm{p}^{32} \mathrm{P}_{1 / 2}$ & $1.93 \mathrm{e}+02$ & & 0 \\
\hline 1356.47 & $\mathrm{H}_{2}$ & 2.37708 & $0-4 R 7 \quad(X-B)$ & & & & 0 \\
\hline 1360.78 & $\mathrm{Al} I \mathrm{IX} / 2$ & 8.19409 & $2 s^{2} 2 p^{2} P_{1 / 2}$ & $-2 \mathrm{~s} 2 \mathrm{p}^{2}{ }^{4} \mathrm{P}_{3 / 2}$ & $3.57 \mathrm{e}+03$ & weak in QS & 0 \\
\hline 1364.87 & $\mathrm{NaV}$ & 1.65732 & $2 s^{2} 2 p^{3}{ }^{4} S_{3 / 2}$ & $-2 s^{2} 2 p^{3}{ }^{2} P_{3 / 2}$ & $4.16 \mathrm{e}+00$ & & 0 \\
\hline 1365.51 & $\mathrm{Na} \mathrm{V}$ & 2.25491 & $2 s^{2} 2 p^{3}{ }^{4} S_{3 / 2}$ & $-2 s^{2} 2 p^{3}{ }^{2} P_{1 / 2}$ & $4.16 \mathrm{e}+00$ & & 0 \\
\hline 1367.12 & S III? & 1.58489 & $3^{s 2} 3 p^{2}{ }^{1} D_{2}$ & $-3^{\mathrm{s} 2} 3 \mathrm{p} 3 \mathrm{~d}^{1} \mathrm{~F}_{3}$ & & & 3 \\
\hline 1371.32 & $\mathrm{Ov}$ & 164.349 & $2 \mathrm{~s} 2 \mathrm{p}^{1} \mathrm{P}_{1}$ & $-2 p^{21} D_{2}$ & $3.36 \mathrm{e}+08$ & & 0 \\
\hline 1372.89 & $\mathrm{Fe}$ VIII/2 & 3.83338 & $3 p^{5} 3 d^{2}{ }^{2} P_{3 / 2}$ & $-3 p^{6} 4 d^{2} D_{5 / 2}$ & $2.73 \mathrm{e}+09$ & & 1 \\
\hline 1382.88 & $\mathrm{Ca} I X / 2$ & 12.1360 & $3^{32}{ }^{1} S_{0}$ & $-3^{\mathrm{s} 3 \mathrm{p} 3} \mathrm{P}_{1}$ & $6.05 \mathrm{e}+05$ & corona & 0 \\
\hline 1385.74 & $?$ & 1.51274 & & & & & 0 \\
\hline 1389.44 & $\mathrm{Si}$ IX/2 & 5.29982 & $2 \mathrm{~s}^{2} 2 \mathrm{p}^{2}{ }^{3} \mathrm{P}_{2}$ & $-2 \mathrm{~s} 2 \mathrm{p}^{3}{ }^{5} \mathrm{~S}_{2}$ & $1.38 \mathrm{e}+05$ & corona & 0 \\
\hline 1394.28 & $\mathrm{Fe} \mathrm{VIII/2}$ & 38.5647 & $3 p^{6} 4 p^{2} P_{1 / 2}$ & $-3 p^{6} 4 d^{2} D_{3 / 2}$ & $8.21 \mathrm{e}+09$ & & 1 \\
\hline 1397.22 & OIV & 20.9203 & $2 \mathrm{~s}^{2} 2 \mathrm{p}^{2} \mathrm{P}_{1 / 2}$ & $-2 \mathrm{~s} 2 \mathrm{p}^{2}{ }^{4} \mathrm{P}_{3 / 2}$ & $3.75 \mathrm{e}+01$ & & 0 \\
\hline 1398.06 & S IV & 2.50008 & $3^{\mathrm{s} 2} 3 \mathrm{p}^{2} \mathrm{P}_{1 / 2}$ & $-3^{s 3 p^{2}{ }^{4}} P_{3 / 2}$ & $1.05 \mathrm{e}+03$ & & 0 \\
\hline 1399.77 & O IV & 91.5729 & $2 s^{2} 2 p^{2} P_{1 / 2}$ & $-2 \mathrm{~s} 2 \mathrm{p}^{2}{ }^{4} \mathrm{P}_{1 / 2}$ & $1.47 \mathrm{e}+03$ & weak in QS & 0 \\
\hline 1400.52 & Ar VIII/2 & 23.3320 & $2 p^{6} 3^{s}{ }^{2} S_{1 / 2}$ & $-2 p^{6} 3 p^{2} P_{3 / 2}$ & $2.73 e+09$ & corona & 0 \\
\hline 1401.16 & O IV & 437.363 & $2 \mathrm{~s}^{2} 2 \mathrm{p}^{2} \mathrm{P}_{3 / 2}$ & $-2 \mathrm{~s} 2 \mathrm{p}^{2}{ }^{4} \mathrm{P}_{5 / 2}$ & $1.16 \mathrm{e}+03$ & weak in QS & 0 \\
\hline 1404.79 & S IV & & $3^{\mathrm{s} 2} 3 \mathrm{p}^{2} \mathrm{P}_{1 / 2}$ & $-3^{\mathrm{s} 3 \mathrm{p}^{2}{ }^{4}} \mathrm{P}_{1 / 2}$ & $6.39 \mathrm{e}+04$ & weak in QS & 0 \\
\hline 1404.82 & O IV & & $2 s^{2} 2 p^{2} P_{3 / 2}$ & $-2 \mathrm{~s} 2 \mathrm{p}^{2}{ }^{4} \mathrm{P}_{3 / 2}$ & $2.90 \mathrm{e}+02$ & weak in QS & 0 \\
\hline 1406.04 & S IV & 30.9620 & $3^{\mathrm{s} 2} 3 \mathrm{p}^{2} \mathrm{P}_{3 / 2}$ & $-3^{\mathrm{s} 3 \mathrm{p}^{2}{ }^{4}} \mathrm{P}_{5 / 2}$ & $5.13 \mathrm{e}+04$ & & 0 \\
\hline 1407.39 & O IV & 80.3204 & $2 s^{2} 2 p^{2} P_{3 / 2}$ & $-2 \mathrm{~s} 2 \mathrm{p}^{2}{ }^{4} \mathrm{P}_{1 / 2}$ & $1.45 \mathrm{e}+03$ & & 0 \\
\hline 1412.10 & $\operatorname{Mg}$ IX/2 & 26.5676 & $2 \mathrm{~s}^{2}{ }^{1} \mathrm{~S}_{0}$ & $-2 \mathrm{~s} 2 \mathrm{p}^{3} \mathrm{P}_{1}$ & $9.04 \mathrm{e}+04$ & & 0 \\
\hline 1413.65 & ? & 2.43612 & & & & & 0 \\
\hline 1416.93 & S IV & 19.6082 & $3^{\mathrm{s} 2} 3 \mathrm{p}^{2} \mathrm{P}_{3 / 2}$ & $-3^{\mathrm{s} 3 \mathrm{p} 2{ }^{4}} \mathrm{P}_{3 / 2}$ & $2.16 \mathrm{e}+04$ & & 0 \\
\hline 1423.86 & S IV & 5.75110 & $3^{\mathrm{s} 2} 3 \mathrm{p}^{2} \mathrm{P}_{3 / 2}$ & $-3^{\mathrm{s} 3 \mathrm{p} 2{ }^{4}} \mathrm{P}_{1 / 2}$ & $4.72 \mathrm{e}+04$ & & 0 \\
\hline 1425.42 & $\mathrm{~S} \mathrm{VI} / 2$ & 11.0943 & $2 p^{6} 3 p^{2} P_{3 / 2}$ & $-2 p^{6} 3 d^{2} D_{5 / 2,3 / 2}$ & $5.20 \mathrm{e}+09$ & & 0 \\
\hline 1426.49 & $\mathrm{H}_{2}$ & 2.76212 & $0-5 P 7 \quad(X-B)$ & & & & 0 \\
\hline 1427.68 & Ar VIII/2 & 9.64131 & $2 p^{6} 3^{s}{ }^{2} S_{1 / 2}$ & $-2 p^{6} 3 p^{2} P_{1 / 2}$ & $2.40 \mathrm{e}+09$ & & 0 \\
\hline 1431.03 & $\mathrm{H}_{2}$ & 3.33829 & $1-6 R 3 \quad(X-B)$ & & & & 2 \\
\hline 1431.32 & $\mathrm{Arv} / 2$ & 2.33605 & $3^{\mathrm{s} 2} 3 \mathrm{p}^{2}{ }^{3} \mathrm{P}_{2}$ & $-3^{\mathrm{s} 3 \mathrm{p} 3{ }^{3} \mathrm{P}_{2,1}}$ & & & 0 \\
\hline
\end{tabular}


H. Tian et al.: Solar transition region above sunspots, Online Material p 6

Table 6. continued.

\begin{tabular}{|c|c|c|c|c|c|c|}
\hline$\lambda_{\text {obs }}(\AA)$ & Line & Intensity & Transition & $A_{\mathrm{ki}}\left(\mathrm{s}^{-1}\right)$ & Also seen in & Reference \\
\hline 1436.52 & (e)/2 & 11.2925 & & & & 0 \\
\hline 1439.11 & $\mathrm{H}_{2}$ ? & 2.37439 & & & & 3 \\
\hline 1442.54 & $\mathrm{Fe}$ VIII/2 & 13.2009 & $3 p^{6} 4 p^{2} P_{3 / 2}-3 p^{6} 4 d^{2} D_{5 / 2}$ & $2.80 \mathrm{e}+00$ & & 1 \\
\hline 1444.09 & $\mathrm{CO}$ & 5.07571 & $5-1 Q 31 \quad(A-X)$ & & & 2 \\
\hline 1445.77 & Si VIII & 16.3185 & $2 s^{2} 2 p^{3}{ }^{4} S_{3 / 2}-2 s^{2} 2 p^{3}{ }^{2} D_{3 / 2}$ & $1.55 \mathrm{e}+00$ & & 0 \\
\hline 1446.12 & $\mathrm{H}_{2}$ & 5.06184 & $1-6 \mathrm{P} 5 \quad(X-B)$ & & & 3 \\
\hline 1453.08 & $\mathrm{H}_{2}$ & 3.71507 & $O-5 \mathrm{P} 10 \quad(X-B)$ & & & 2 \\
\hline 1458.14 & $?$ & 3.33749 & & & weak in QS & 0 \\
\hline 1477.77 & $\mathrm{Fe}$ VII/2 & 41.6691 & $3 d 4 p{ }^{3} P_{2} \quad-3 d 4 d{ }^{3} P_{2}$ & & & 1 \\
\hline 1480.22 & Ar VIII/2 & 179.944 & $2 p^{6} 4 d^{2} D_{5 / 2}-2 p^{6} 5 p^{2} P_{3 / 2}$ & & & 0 \\
\hline 1482.36 & $\mathrm{Fe} \mathrm{VII/2}$ & 37.2067 & $3 \mathrm{~d} 4 \mathrm{p}^{3} \mathrm{P}_{1} \quad-3 \mathrm{~d} 4 \mathrm{~d}^{3} \mathrm{P}_{0}$ & & & 1 \\
\hline
\end{tabular}

